\title{
Congresos de Anatomía del Cono Sur. 21 años de Historia
}

\author{
Congresses of Anatomy of the Southern Cone. 21 Years of History
}

Mariano del Sol ${ }^{1,3,4} \&$ Ricardo J. Losardo ${ }^{2,3,4,5}$

\begin{abstract}
DEL SOL, M. \& LOSARDO, R. J. Congresos de Anatomía del Cono Sur. 21 años de historia. Int. J. Morphol., 38(3):689-705, 2020.
RESUMEN: Realizamos una reseña histórica de los Congresos de Anatomía del Cono Sur. A inicios del año 1999, se estableció un acuerdo entre la "Sociedad Chilena de Anatomía" y la "Asociación Rioplatense de Anatomía" para realizar -de manera conjunta- los congresos científicos de ambas sociedades. Unos meses después, también a propuesta de los Dres. Mariano del Sol y Ricardo Losardo, se solicitó que se realizara una nueva entidad a la cual denominaron inicialmente "Congreso de Anatomistas del Cono Sur", cuya finalidad sería reunir no solamente a los anatomistas argentinos, uruguayos y chilenos, sino también incorporar a los morfólogos de otros países de América del Sur, entre los cuales estaban los brasileños y los paraguayos. De esta manera, se inició la realización conjunta de los tres congresos (Chileno, Rioplatense y del Cono Sur) con una masiva participación de los morfólogos de numerosos países de nuestro continente. Los "Congresos de Anatomía del Cono Sur" (llamados así desde el II Congreso realizado en Chile) se han realizado normalmente cada año, muchas veces de manera conjunta con los Congresos Argentinos, Chilenos, Brasileños y Panamericanos de Anatomía, entre otros eventos. Al cumplirse 21 años del inicio de esta fructífera y beneficiosa unión científica quisimos plasmar en este artículo los orígenes de estos Congresos de Anatomía del Cono Sur, como también los motivos que llevaron a su creación. Asimismo, se enumeraron los encuentros realizados en los primeros 21 años (1999-2019), señalando sus fechas, sedes y presidentes.
\end{abstract}

PAlABRAS ClAVE: Anatomía; Congreso de Anatomía del Cono Sur; Historia de la Anatomía; Sociedades de Anatomía.

\section{INTRODUCIÓN}

Los Congresos de Anatomía del Cono Sur nacieron con el objetivo principal de fomentar la relación entre la Sociedad Chilena de Anatomía y la Asociación Rioplatense de Anatomía (Argentina-Uruguay) y mejorar la comunicación entre los asociados. Los mentores de esa iniciativa fueron los Prof. Dres. Mariano del Sol y Ricardo Losardo, quienes ya habían concretado otras acciones de acercamiento entre ambas sociedades con tal fin.

El I Congreso de Anatomistas del Cono Sur se realizó en la ciudad de Mendoza, Argentina, en 1999; y el XXI Congreso de Anatomía del Cono Sur en Pucón, Chile, en 2018, ambas ciudades trasandinas; una manera de demostrar que la cordillera de los Andes, en vez de separar, une a los países que la comparten.

\section{Los inicios}

A inicios de 1999 en una reunión de la Directiva de la Asociación Rioplatense de Anatomía realizada en la ciu- dad de La Plata, el Prof. Dr. Ricardo Losardo le comunicaba a sus colegas que el Prof. Dr. Mariano del Sol, Académico de la Facultad de Medicina de la Universidad de La Frontera, Chile, le había planteado la posibilidad de unir los Congresos de Anatomía realizados por la Asociación Rioplatense de Anatomía y la Sociedad Chilena de Anatomía; y además, realizar estos congresos junto al naciente Congreso de Anatomistas del Cono Sur y proponer que ellos se realizaran en Mendoza, ciudad argentina, donde se llevaría a cabo ese año, el XXXVI Congreso de la Asociación Rioplatense de Anatomía.

Debido a la incipiente red de correo electrónico, aún la comunicación se realizaba vía Fax, telefónica o incluso por carta vía correo postal. Una vez establecido el contacto entre los colegas organizadores de los Congresos, el Dr. Mariano del Sol se comunicó con la Presidente del Congreso Rioplatense de Anatomía, Prof. Dra. Alba Suárez de Rossi, para afinar detalles de los congresos conjuntos y se decidió hacer reuniones con la participación de ambos presidentes y

\footnotetext{
${ }^{1}$ Centro de Excelencia en Estudios Morfológicos y Quirúrgicos (CEMyQ), Facultad de Medicina, Universidad de La Frontera (UFRO), Temuco, Chile.

${ }^{2}$ Último Presidente de la Asociación Rioplatense de Anatomía (ARA) y Presidente fundador de la Asociación Argentina de Anatomía (AAA).

${ }^{3}$ Ex Presidente de la Asociación Panamericana de Anatomía.

${ }^{4}$ Co Presidente de la Academia Panamericana de Anatomía.

${ }^{5}$ Escuela de Graduados, Facultad de Medicina, Universidad del Salvador (USAL), Buenos Aires, Argentina.
} 
del Prof. Dr. Ricardo Jorge Losardo, Vice presidente de la Asociación Rioplatense de Anatomía y uno de los principales mentores de este estrecho vínculo argentino-chileno; y que había participado tantas veces en proyectos conjuntos, para ser más precisos, desde el mes de octubre de 1989, cuando se realizó el X Encuentro Nacional de Anatomistas de Chile en la ciudad de Concepción, Chile; evento que pasó posteriormente, a propuesta del Prof. Dr. Mariano del Sol, a denominarse Congreso Chileno de Anatomía, a partir de 1990.

Se hicieron reuniones previas para armar el Congreso conjunto; y los Dres. Losardo y del Sol se movilizaron hasta Mendoza. Se desarrollaron principalmente en las dependencias de la Cátedra de Anatomía Normal de la Facultad de Ciencias Médicas, de la Universidad Nacional de Cuyo y de la Universidad del Aconcagua, en Mendoza; pero incluso en oportunidades hasta en los salones del aeropuerto de la misma ciudad ya que se combinaba la hora de la llegada de los Dres. Losardo y del Sol desde Buenos Aires y de Temuco - Santiago, respectivamente. En dos ocasiones, estas reuniones duraron entre 2 a 3 horas, ya que los Dres. Losardo y del Sol debían tomar el avión de regreso a Buenos Aires y a Temuco.

El día 9 de abril de 1999, ya numerosos aspectos de los Congresos habían sido consensuados y se determinó que el I Congreso de Anatomistas del Cono Sur, el XXXVI Congreso de la Asociación Rioplatense de Anatomía y el XX Congreso Chileno de Anatomía, se realizarían en la ciudad de Mendoza, entre los días 13 y 15 de octubre de 1999.

\section{Acuerdo entre la "Sociedad Chilena de Anatomía" y la "Asociación Rioplatense de Anatomía".}

Con fecha 9 de abril de 1999, la Sociedad Chilena de Anatomía comunicó a la Secretaría de la Asociación Rioplatense de Anatomía en Buenos Aires, el acuerdo para la realización de los Congresos Chileno y Rioplatense de Anatomía, de manera conjunta entre ambas sociedades.

El día 16 de abril de 1999 vía Fax se le comunicó e informó a la Prof. Dra. Alba Suárez de Rossi, Presidente del Comité Ejecutivo Organizador del XXXVI Congreso de la Asociación Rioplatense de Anatomía, el acuerdo de la Sociedad Chilena de Anatomía de realizar el Congreso de manera conjunta y también sobre la constitución del Comité Organizador del XX Congreso Chileno de Anatomía.

Nuevas y numerosas comunicaciones, vía telefónica con Argentina (Buenos Aires y Mendoza) fueron realizadas, como también se establecieron contactos vía Fax, correo ordinario y reuniones conjuntas en la ciudad de Mendoza. En estas reuniones participaron además de los
Presidentes de los Congresos Chileno y Rioplatense de Anatomía, el Prof. Dr. Ricardo Losardo de la Directiva de la "Asociación Rioplatense de Anatomía" quien viajó desde Buenos Aires a Mendoza.

El día 14 de mayo de 1999, se estableció un acuerdo entre la Sociedad Chilena de Anatomía y la Asociación Rioplatense de Anatomía para la realización de este primer evento, quedando establecido, que:

a) El Programa Científico del Congreso sería realizado en forma conjunta por representantes de ambas Sociedades. La participación científica sería de un 50\% para cada Sociedad (invitados, conferencias y cursos).

b) Cada Sociedad inscribiría a sus socios en sus respectivas sedes. El monto de esta recaudación pasaría a cada una de las Sociedades. Ninguna de ellas debería dar explicaciones a la otra sobre los montos recaudados.

c) Los gastos (viajes, estadías, etc.) de los conferencistas extranjeros invitados por una u otra Sociedad estarían a cargo de la Sociedad que invita.

d) La Asociación Rioplatense de Anatomía (A.R.A.) manejaría la tesorería del Congreso, haciéndose cargo de las ganancias y/o pérdidas del Congreso.

e) La Sociedad Chilena de Anatomía se desvincularía (del control y/o supervisión) de la tesorería del Congreso.

f) La Sociedad Chilena de Anatomía entregaría un listado con los miembros que estarían en condiciones de ingresar al Congreso.

g) La Sociedad Chilena de Anatomía debería publicar en la Revista Chilena de Anatomía los resúmenes de los trabajos presentados en el Congreso. Aquellos trabajos inscritos en el Congreso y que no fuesen presentados no serían publicados. La publicación aparecería con posterioridad al Congreso (manteniendo las fechas habituales de impresión). La Sociedad Chilena de Anatomía entregaría sin costo un ejemplar a cada Miembro Titular inscrito en el Congreso (Argentino). Las revistas serían entregadas en la sede administrativa de la Asociación Rioplatense de Anatomía, que se encargaría de la distribución posterior a sus socios. Los resúmenes podrían ser modificados por el Comité Científico de la Revista, respecto a Nomenclatura Anatómica Internacional y otros detalles que estimaba conveniente (sin aviso previo a los autores).

Firmaron este acuerdo los Profesores Dres. Fernando Matamala V., Presidente de la Sociedad Chilena de Ana- 
tomía; Enrique Olave R., Secretario General de la Sociedad Chilena de Anatomía; Ricardo J. Losardo, Vice-Presidente de la Asociación Rioplatense de Anatomía; Andrea Prina, Tesorera de la Asociación Rioplatense de Anatomía; y Mariano del Sol C., Presidente del XX Congreso Chileno de Anatomía.

Como resultado fue aceptada la iniciativa de los Dres. Mariano del Sol y Ricardo Losardo quienes plantearon la creación y realización de un nuevo Congreso, más amplio y con mayor participación internacional que denominaron "Congreso de Anatomistas del Cono Sur", que uniría no solamente a los anatomistas argentinos, chilenos y uruguayos sino también a los colegas brasileños y paraguayos.

\section{Primer Congreso de Anatomistas del Cono Sur (Mendoza, Argentina)}

El Primer Congreso de Anatomistas del Cono Sur se hizo entre el 13 y el 15 de octubre de 1999 y se realizó en el "Centro de Congresos y Exposiciones" de la ciudad de Mendoza (Fig. 1). Se habilitaron ocho salas y el aula magna, lo que demostró la intensa actividad científica y la numerosa concurrencia.

La mayor parte de los más de 120 asistentes chilenos al Congreso cruzaron la Cordillera de los Andes por tierra, desde distintas partes de Chile. Lo hicieron preferentemente en auto, dada la cercanía de Santiago con Mendoza. Sin embargo, hubo morfólogos de Puerto Montt por el Sur y Arica por el extremo norte del país, que lo hicieron en avión o en bus hasta Santiago y de ahí a Mendoza por tierra. En ese tiempo los recursos audiovisuales eran escasos y caros; y como en Mendoza no tenían suficientes para todas las ac-

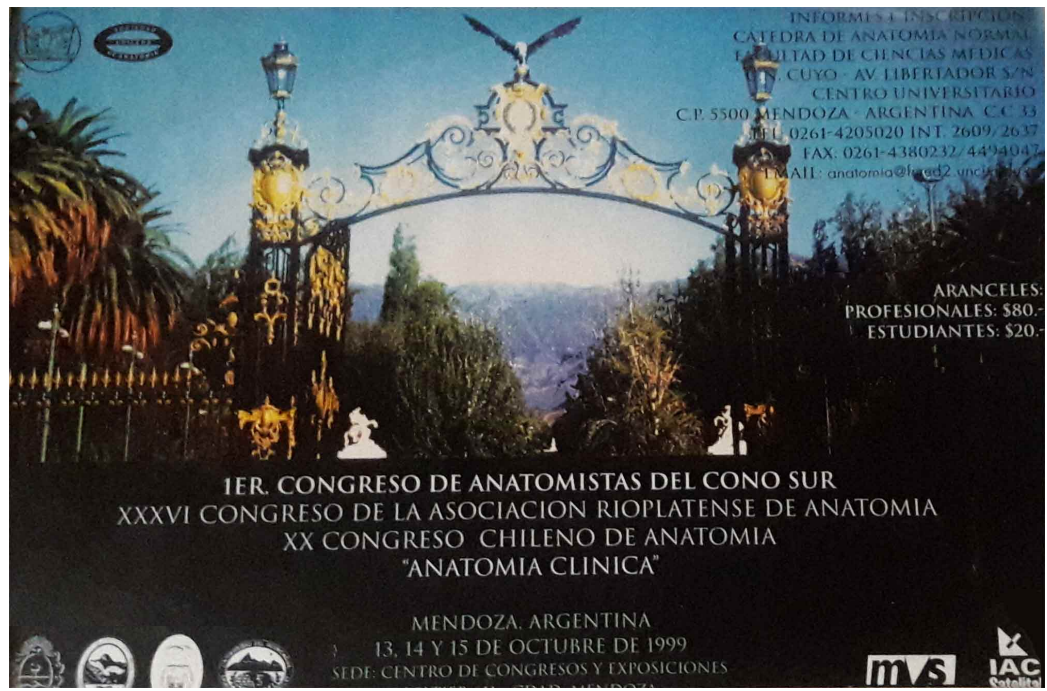

Fig. 1. Poster del I Congreso de Anatomía del Cono Sur, realizado en la ciudad de Mendoza, Argentina. tividades que se realizarían, desde Temuco fueron llevados proyectores de diapositivas (slides) y también proyectores portátiles (Data Show), aparatos que en esa época eran muy costosos y extremadamente grandes y pesados (por supuesto aún no existían los LED). Por lo anterior, se tuvo que hacer trámites aduaneros y de permisos universitarios para poder sacarlos del país. Algo impensado en estos días.

El Comité Ejecutivo Organizador del Congreso de Anatomistas del Cono Sur estuvo conformado de la siguiente manera: a) Por parte de la Sociedad Chilena de Anatomía: Presidente: Mariano del Sol C., Vice Presidente: Alberto Rodríguez T. Secretario: Enrique Olave R. Tesorera: Daisy Cabalín Silva. Comité Científico: Presidente: Enrique Olave R. Integrantes: Marta Bretos A., Hermes Bravo, Humberto Guiraldes del Canto, Mariana Rojas R., Atilio Almagiá F., María Angélica Montenegro R. y Alberto Rodríguez Torres. Relaciones Internacionales: Fernando Matamala V. Secretaría Administrativa: Sandra Villa Baier y Vivian Cullen F. b) Por parte de la Asociación Rioplatense de Anatomía (Argentina - Uruguay): Presidente: Alba E. Suárez de R. Vice Presidente: Elia Martino de V. Secretario: Juan C. Torcivia. Tesorero: Luis A. Rivamar. Comité Científico: Carlos A. La Rocca, Juan C. Luciarte, Roberto M. Martínez, Aldo G. Molina y Marta Souto. Comité Foro Estudiantil: Luis D. Calderón. Relaciones Públicas e Internacionales: Adriana B. Rossi. Secretaría Administrativa: María E. Britos y Pablo Rossi. Hubo un jurado de premios constituido por: Salomón Farache, Alberto Fontana, Alberto Poli, Antonio Méndez Anell y Conrado Valvo.

La conferencia inaugural del I Congreso de Anatomistas del Cono Sur estuvo a cargo del Prof. Dr. Roberto M. Aprá, ex Profesor Titular de «Clínica Médica» de la Facultad de Ciencias Médicas de la Universidad Nacional de Cuyo, Mendoza; y Profesor Titular de «Atención al Enfermo II» de la Facultad de Ciencias de la Salud de la Universidad de Mendoza.

El Prof. Aprá presentó una visión de la Anatomía al final del segundo milenio, señalando "Desde que la anatomía se constituyó en ciencia cabal se ha hecho tan abarcativa que, en contraste con su precisión epistemológica, su papel actual y futuro es un interrogante motivador dentro de las disciplinas que tratan de las ciencias relacionadas con la vida". Termina señalando: "La Anatomía nos ha conquistado verdades irrebatibles, y constituye uno de los terrenos más firmes de la ciencia médica". "Los anatomistas nos han enseñado, a partir 
del Herófilo, Erasístrato, Galeno y Vesalio, a hacer ciencia con objetividad. Y, por último, pensando en el futuro, nada en las Ciencias de la Salud podrá desvincularse de la Anatomía, por más original que sea la investigación. Como en las preguntas socráticas, siempre habrá que referirse a un "dónde" para que la respuesta sea completa. La Anatomía será raíz y fundamento, y será esencia con una noble misión: Existir, y sólo existir, para el hombre y para la Humanidad".

Durante el Congreso se realizó un homenaje al Prof. Dr. Guillermo Saredo, médico veterinario y Profesor de la Universidad de Buenos Aires, que fue Presidente de la Asociación Rioplatense de Anatomía (1995-1996) y que había fallecido el 8 de agosto de 1999.

También durante este Congreso, la Asociación Rioplatense de Anatomía, instauró el Premio Anual "Alfonso Roque Albanese" al mejor trabajo realizado por estudiantes, con el fin de estimular la investigación anatómica desde los inicios de la formación, y fomentar el concepto que la enseñanza de la medicina debe ser integral (Losardo et al., 2017). El jurado del premio estuvo constituido por los Dres. Eduardo Albanese (hijo menor), Ricardo Losardo y Diana Perriard. Tuvo la particularidad que el Maestro Albanese, nonagenario, estuvo presente en la exposición de los trabajos que optaron a este premio. El Maestro donó una suma de 100 dólares para el trabajo premiado y esto continuó así hasta su fallecimiento.

El Congreso contó con morfólogos argentinos, chilenos y uruguayos, además se cumplió el objetivo principal de lograr la participación de los brasileños, quienes en un número superior a 50 inscritos, presentaron 22 trabajos de investigación realizados en diversas universidades brasileñas y de diferentes Estados, entre los cuales podemos destacar a investigadores de San Pablo, Minas Gerais, Santa Catarina y Río de Janeiro. Incluso se contó con la participación de tres destacados conferencistas brasileños, dos de ellos de la Universidade do Estado de Rio do Janeiro, los Profs. Dres. Carlos Mandarim de Lacerda y Henrique Ayres Vasconcellos; y el tercero, de la Universidade Federal de São Carlos, SP, el Prof. Dr. Francisco Martínez, quien participó con la charla "Contribución de la morfología a la biología de la reproducción", ampliando así la temática del Congreso. El Maestro José Carlos Prates, asiduo asistente a los Congresos Rioplatenses y Chilenos también estuvo presente y aportó sus apreciados conocimientos. Fue destacada la participación durante todo el congreso del Prof. Dr. Daniel Alonso, mendocino radicado en Estados Unidos de Norteamérica, de la Universidad de Cornell - Nueva York, que dictó una interesante conferencia sobre "La enseñanza de la anatomía en Cornell".
Finalmente, el día 21 de octubre se remitió a cada uno de los profesores invitados, una carta de agradecimiento por parte del Comité Organizador del "I Congreso de Anatomistas del Cono Sur, XXXVI Congreso de la Asociación Rioplatense de Anatomía y XX Congreso Chileno de Anatomía", agradeciendo su participación fundacional en este evento.

El I Congreso de Anatomía del Cono Sur fue todo un éxito y una experiencia académica y de investigación nueva que se replicaría al año siguiente en Chile.

\section{Segundo Congreso de Anatomía del Cono Sur (Santia- go, Chile)}

A partir del segundo congreso, éste cambió ligeramente su nombre a "Congreso de Anatomía del Cono Sur", para de esta manera, incluir además a los colegas de las otras áreas de las ciencias morfológicas, en especial, a los histólogos y embriólogos como también a los profesores e investigadores de ciencias morfológicas veterinarias incluyendo a la morfología experimental.

El II Congreso de Anatomía del Cono Sur se realizó entre el 2 y el 4 de noviembre de 2000 en la Universidad Iberoamericana de Ciencias y Tecnología (UNICIT), de la ciudad de Santiago, que también tuvo una importante actividad científica y una numerosa concurrencia (Fig. 2).

El Comité Ejecutivo Organizador del II Congreso de Anatomía del Cono Sur estuvo conformado de la siguiente manera:

a) Por parte de la Sociedad Chilena de Anatomía: Presidente: Hermes Bravo C., Vice Presidente: Eduardo BustosObregón, Secretaria: Mariana Rojas R., Tesorero: Oscar Inzunza H. Comité Científico Presidente: Alberto Rodríguez T. Integrantes: Mariana Rojas R., María Angélica Montenegro R., Susana Domínguez V., Cleofina Bosco B., Fernando Matamala V., Eduardo D'Acuña U., David Lemus A., Atilio Almagiá F. y Mariano del Sol C., Relaciones Públicas e Internacionales: Eduardo Bustos-Obregón. Secretaría Administrativa: Sandra Villa B. y Vivian Cullen F.

b) De parte de la Asociación Rioplatense de Anatomía (Argentina - Uruguay): Presidente: Ricardo Losardo, Vice Presidente: Alberto Fontana, Secretario: Inés Castellano, Tesorera: Andrea Prina. Comité Científico: Jorge Binetti, Raúl M. Lagraña, Juan C. Miguel, Diana Perriard, Adalberto Ruiz y Eduardo A. Spinelli. Hubo un Jurado de Premios constituido por Juan C. Barrovecchio, Alberto Fontana, Rodolfo Lafalla, Ricardo Jorge Losardo, Antonio Méndez Anell y Diana Perriard. 


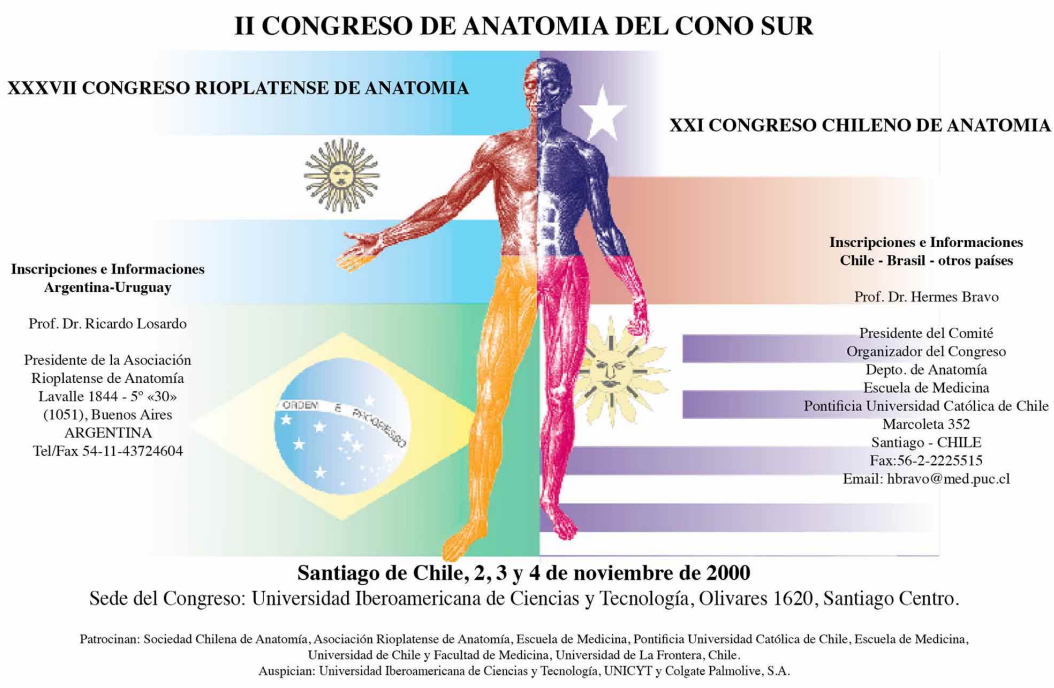

Fig. 2. Poster del II Congreso de Anatomía del Cono Sur, realizado en la ciudad de Santiago de Chile.

El congreso fue inaugurado por el Rector de UNICIT, el Prof. Dr. Fernando Monckeberg Barros. Se destacaron las conferencias brindadas por los Prof. Dres. José Luis Velayos, de la Universidad Autónoma de Madrid, España, una sobre docencia en anatomía y otra sobre anatomía del insomnio; Gerardo Medeiros de la Fundação Lusiada, Brasil, sobre historia de la anatomía; Oscar Rocha Barbosa de la Universidade do Estado de Rio de Janeiro, Brasil, sobre biomecánica; y Michel Guillomot de French National Institute for Agricultural Research, París, Francia, sobre desarrollo embriológico.

También se destacó la mesa redonda sobre Nomenclatura Anatómica, coordinada por el Prof. Dr. Mariano del Sol y con la participación de los internacionalmente reconocidos expertos en el tema, Prof. Dres. Rolando Cruz Gutiérrez, de Costa Rica; José Carlos Prates, de Brasil; y Alberto Rodríguez Torres, de Chile; quienes constituirían algunos años después los pilares principales del SILAT (Cruz-Gutiérrez et al., 2010a).

Por otra parte, el curso dictado por el Prof. Dr. César Herminio Bergottini y su equipo de Corrientes, Argentina, sobre "Introducción a la investigación científica. Un enfoque epístemo-metodológico", se extendió a todos los días del Congreso y contó con una numerosa y entusiasta asistencia estudiantil.

El discurso de clausura del II Congreso de Anatomía del Cono Sur estuvo a cargo del Prof. Dr. Eduardo Bustos Obregón, Profesor Titular de la Facultad de Medicina de la Universidad de Chile, destacando que "lo ecuménico, en un Congreso que contó con la presencia de tres Universidades para su gestión e implementación, una de ellas privada
(UNICIT) junto a la Pontificia Universidad Católica y la Universidad de Chile. El Comité Científico estuvo conformado por destacados especialistas de Chile y Argentina; y contó con el apoyo irrestricto de nuestros colegas de la Universidad de La Frontera". Señaló también: "En un muro del Pabellón de Microscopía del Instituto de Anatomía de la Universidad de Hamburgo (Alemania), donde tuve el privilegio de trabajar con una beca de investigación de la Fundación Alexander von Humboldt hace casi 30 años, se lee : «Was ist das Schwerste von Allem was Dir das Leichteste duenket mit den Augen zu sehen was vor den Augen Dir liegt» (Lo más difícil de todo, aunque pienses que es lo más fácil, es ver verdaderamente con los ojos lo que está frente a ellos)".

También Bustos-Obregón destacó: “Lo promisorio, con la continuidad de estos eventos internacionales del Cono Sur, que posibilitan nuestro diálogo horizontal entre colegas de países hermanos. Sobre todo, la participación numerosa de alumnos, muchos de ellos futuros profesionales de la Salud, para quienes el marco de su profesión no solo no los limita, sino que los impele al cultivo del conocimiento y la investigación en Ciencias Morfológicas. Como única recompensa aspiran (en palabras adaptadas de Laín Entralgo) a «ascender a la difícil cumbre de las ciencias para desde allí (como un montañista remonta peligrosas cimas) contemplar desde otra perspectiva el valle».

Se repitió de esta manera el éxito del primer congreso tanto en la parte científica -con más de 200 trabajos científicos presentados- como en la numerosa asistencia de profesores de los países pertenecientes al Cono Sur de América.

\section{FUNDACIÓN Y OBJETIVOS}

La idea original era realizar un congreso, un año, en Chile; y el otro, en Argentina-Uruguay. De esta manera, cada congreso del Cono Sur acompañaría sucesivamente a un congreso chileno y a uno rioplatense.

La finalidad era asegurar una concurrencia masiva de profesores y alumnos de ambas sociedades anatómicas. El programa científico se organizaba de manera compartida, quedando el programa social y cultural a cargo de la sociedad que tenía la sede. Ambas sociedades disponían de un espacio para tratar y resolver sus temas administrativos. Además, se disminuían los gastos que tenían que afrontar cada sociedad en la organización de su congreso anual. Por 
otra parte, se mantenían los costos de inscripción que cada sociedad tenía y resultaban por cierto bajos para el valor de un congreso internacional.

En 2002 se agregó la Sociedad Brasileña de Anatomía (SBA) y así estos congresos se realizaron con la sociedad de anatomía más extensa y numerosa de América del Sur. Gracias a la entonces Directiva de la SBA con la presidencia del Prof. Dr. Serafim Vicenzo Cricenti y la Secretaría General de la Prof. Dra Nadir Eunice Valverde Barbato de Prates, se logró que en el XX Congreso Brasileño, a celebrarse en Maceió, del 6 al 11 de octubre de 2002, con la presidencia del Prof. Dr. Celio Fernando de Sousa Rodrígues, se uniera por primera vez al Congreso de Anatomía del Cono Sur. Fue el primer congreso donde participaban las tres sociedades: Chilena (Presidente Prof. Dr. Enrique Olave), Argentina (Presidente Prof. Dr. Raúl Lagraña) y Brasilera de Anatomía (Presidente Prof. Dr. Serafim Vincenzo Cricenti); y coincidió con el $50^{\circ}$ aniversario de la SBA. Fueron importantes las gestiones de los Dres. Mariano del Sol y Ricardo Losardo. para lograr esta unión entre las tres Sociedades.

Los concurrentes a estos congresos de Anatomía del Cono Sur tenían la oportunidad de estrechar vínculos entre sí y facilitar el intercambio científico. Así surgieron trabajos multicéntricos, entre distintas universidades de los países participantes; como también, la posibilidad de visitar centros anatómicos universitarios para realizar rotaciones de perfeccionamiento.

\section{CONGRESOS REALIZADOS}

En estos 21 años, los congresos del Cono Sur se realizaron en forma ininterrumpida, venciendo todas las dificultades regionales que caracterizan a estas latitudes y "aggiornandose" a las vicisitudes que se presentaban.

I Congreso de Anatomistas del Cono Sur, XXXVI Congreso de la Asociación Rioplatense de Anatomía y XX Congreso Chileno de Anatomía, en Mendoza, Argentina, durante los días 13, 14 y 15 de octubre de 1999. Presidentes: Prof. Dr. Mariano del Sol C. (Chile) y Prof. Dra. Alba Suárez de Rossi (Argentina). (Sociedad Chilena de Anatomía \& Asociación Rioplatense de Anatomía, 2000). En la página Web se corrigió a I Congreso de Anatomía del Cono Sur.

II Congreso de Anatomía del Cono Sur, XXI Congreso Chileno de Anatomía y XXXVII Congreso Rioplatense de Anatomía, en Santiago de Chile, durante los días 3, 4 y 5 de noviembre de 2000. Presidentes: Profs. Dres. Hermes Bravo (Chile) y Ricardo Losardo (Argentina). Fue el último Congreso con la denominación "Rioplatense". (Sociedad Chilena de Anatomía \& Asociación Rioplatense de Anatomía, 2001).
III Congreso de Anatomía del Cono Sur, XXXVIII Congreso Argentino de Anatomía y XXII Congreso Chileno de Anatomía, I Reunión de Terminología Anatómica en América Hispana, en Buenos Aires, durante los días 22, 23, 24 y 25 de octubre de 2001. Presidentes: Profs. Dres. Mariano del Sol C. (Chile) y Ricardo J. Losardo (Argentina). (Sociedad Chilena de Anatomía \& Asociación Argentina de Anatomía, 2002).

IV Congreso de Anatomía del Cono Sur, XX Congresso Brasileiro de Anatomia, XXIII Congreso Chileno de Anatomía, XXXIX Congreso Argentino de Anatomía y Primer Simpósio sobre Ensino de Anatomia, en Maceió, Brasil, durante los días 6, 7, 8, 9, 10 y 11 de octubre de 2002. Presidente de los congresos: Prof. Dr. Célio Fernando de Sousa Rodrigues (Brasil). (Sociedad Chilena de Anatomía \& Asociación Argentina de Anatomía, 2003).

V Congreso de Anatomía del Cono Sur, XXIV Congreso Chileno de Anatomía, XL Congreso Argentino de Anatomía, en Temuco, Chile, durante los días 12, 13, 14, 15 y 16 de noviembre de 2003. Presidente de los congresos Prof. Dr. Mariano del Sol C. (Chile). (Sociedad Chilena de Anatomía \& Asociación Argentina de Anatomía, 2004).

VI Congreso de Anatomía del Cono Sur, XV Congreso Panamericano de Anatomía, XXI Congresso Brasileiro de Anatomia, XXV Congreso Chileno de Anatomía, XLI Congreso Argentino de Anatomía, II Simpósio sobre Ensino de Anatomia, V Simpósio da Associação Paranaense para o Desenvolvimento do Ensino da Ciência, Simpósio de Biologia do Desenvolvimento - $S B B C$, en Foz de Iguazú, Brasil y Puerto Iguazú, Argentina, durante los días 24, 25, 26, 27 y 28 de octubre de 2004. Presidente de los Congresos Brasileños: Prof. Dr. José Geraldo Calomeno (Brasil); Presidente del Congreso Panamericano: Prof. Dr. Ricardo J. Losardo (Argentina); y Presidente de los Congresos del Cono Sur y Chileno: Prof. Dr. Mariano del Sol C (Chile). (Sociedad Chilena de Anatomía \& Asociación Argentina de Anatomía, 2005). Una delegación de congresistas chilenos se observa en las cataratas del Iguazú (Fig. 3).

VII Congreso de Anatomía del Cono Sur y XXVI Congreso Chileno de Anatomía, en Santiago de Chile, durante los días 12, 13, 14 y 15 de octubre de 2005. Presidente: Prof. Dra. Marcela Fuenzalida (Chile). (Sociedad Chilena de Anatomía, 2006)

VIII Congreso de Anatomía del Cono Sur, XXVII Congreso Chileno de Anatomía y XXII Congresso Brasileiro de Anatomia, en Florianópolis, Brasil, durante los días 8, 9, 10, 11 y 12 de octubre 2006. Presidente del Congreso Brasileño: Prof. Dr. Ademar de Souza (Brasil); Presidente de los Con- 


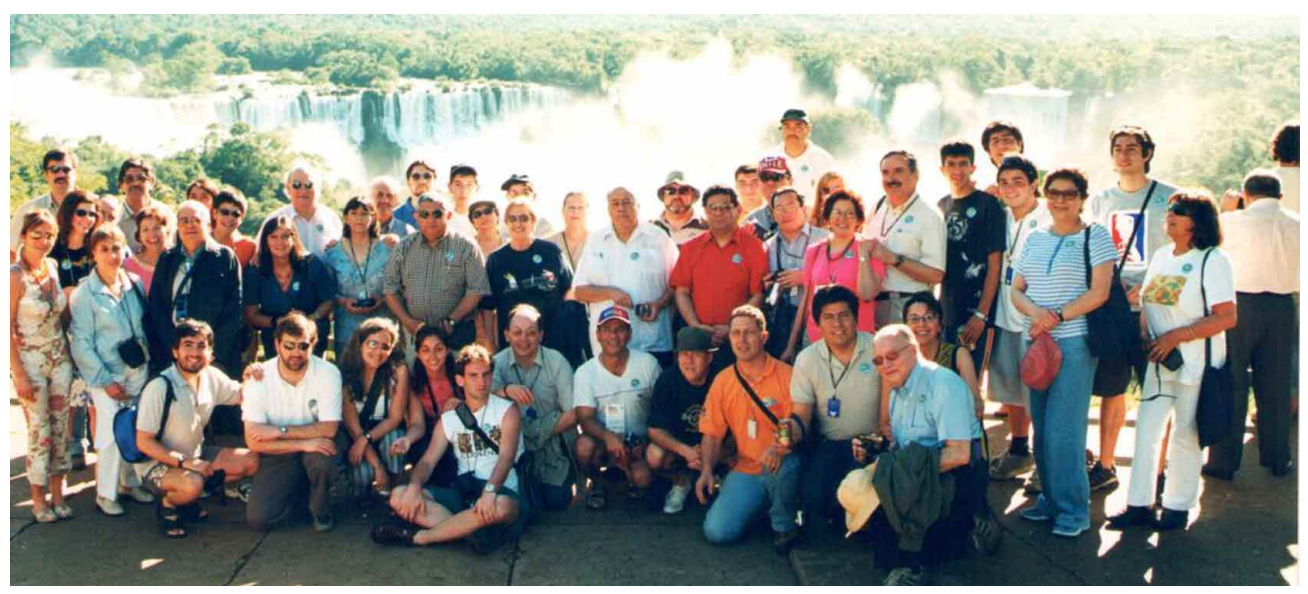

tomía y V Simposio Ibero Latinoamericano de Terminologia Anatomica, Histologica y Embryologica (SILAT V), en Temuco, Chile, durante los días 25, 26, 27, 28, 29 y 30 de octubre de 2010. Presidente: Prof. Dr. Mariano del Sol (Chile). (Sociedad Chilena de Anatomía, 2010).

Fig. 3. Delegación chilena participante del VI Congreso de Anatomía del Cono Sur realizado en Foz de Iguazú en Foz de Iguazú, Brasil y Puerto Iguazú, Argentina, durante los días 24, 25, 26, 27 y 28 de octubre de 2004.

gresos del Cono Sur y Chileno: Prof. Dr. Mariano del Sol (Chile), quien además era el Presidente de la Sociedad Chilena de Anatomía. Cabe destacar que "in memoriam" se nombró Presidente de Honra de este evento a la Profa. Dra. Cristina Ioshie Mizusaki, destacada odontóloga y morfóloga brasileña, profesora de la Universidade Federal de Santa Catarina, quien falleció tempranamente y que desde los inicios participaba de los Congresos de Anatomía del Cono Sur. La Comisión Científica del Congreso de Anatomía del Cono Sur y del Congreso Chileno de Anatomía fueron responsables por el análisis de los Resúmenes de los trabajos científicos presentados en la Sesión de Paneles. Mientras los trabajos de los morfólogos brasileños fueron recibidos y evaluados por la Comisión Científica del XXII Congresso Brasileiro de Anatomia.

IX Congreso de Anatomía del Cono Sur y XXVIII Congreso Chileno de Anatomía en Talca, Chile, durante los días 29, 30 y 31 de octubre de 2007. Presidente: Prof. Dr. Iván Suazo Galdames (Chile).

X Congreso de Anatomía del Cono Sur, XXIII Congresso Brasileiro de Anatomia, XI Congresso Luso-Brasileiro de Anatomia y XXVIII Congreso Chileno de Anatomía, en Belém, Pará, Brasil, durante los días 19, 20, 21, 22 y 23 de octubre de 2008. Presidente del Congreso Brasileño: Prof. Dr. Robson José de Sousa Domingues (Brasil); y de los Congresos del Cono Sur y Chileno: el Prof. Dr. Mariano del Sol (Chile).

XI Congreso de Anatomía del Cono Sur y XXVIII Congreso Chileno de Anatomía, en Santiago de Chile, durante los días 14, 15, 16 y 17 de octubre de 2009. Presidente: Prof. Dr. Juan Carlos López Navarro (Chile).

XII Congreso de Anatomía del Cono Sur, XVII Congreso Panamericano de Anatomía, XXXI Congreso Chileno de Ana-
XIII Congreso de Anatomía del Cono Sur, XXXII Congreso Chileno de Anatomía y el VII Congreso Colombiano de Morfología, en Santa Marta, Colombia, durante los días 27, 28 y 29 de octubre de 2011. Presidente de los Congresos del Cono Sur y Chileno: Prof. Dr. Mariano del Sol (Chile) y Presidente del Congreso Colombiano: Dr. Gary Linero Cueto (Colombia). (Sociedad Chilena de Anatomía, 2012). El Congreso del Cono Sur estuvo patrocinado por la Asociación Panamericana de Anatomía, la Sociedad Chilena de Anatomía y la Asociación Argentina de Anatomía. Fue el primer Congreso del Cono Sur que se hizo fuera de su ámbito geográfico. A los participantes argentinos en una ceremonia organizada por la directiva del Congreso del Cono Sur y en presencia de los participantes de las delegaciones de Argentina y de Chile se les hizo entrega a cada uno de los presentes los certificados y reconocimientos respectivos.

XIV Congreso de Anatomía del Cono Sur, XXXIII Congreso Chileno de Anatomía, XXV Congresso Brasileiro de Anatomia y I Encontro das Ligas Estudantis de Morfologia, en Salvador, Bahía, Brasil, durante los días 3, 4, 5 y 6 de octubre de 2012. Presidente del Congreso Brasileño: Prof. Dr. Atson Carlos de Souza Fernandes (Brasil) y Presidente de los Congresos del Cono Sur y Chileno: Prof. Dr. Mariano del Sol (Chile).

XV Congreso de Anatomía del Cono Sur y XXXIV Congreso Chileno de Anatomía, en Talca, Chile, durante los días 20, 21, 22 y 23 de noviembre de 2013. Presidente: Prof. Dr. Claudio Cruzat Cruzat (Chile). (Campus tv Universidad de Talca, 2013).

XVI Congreso de Anatomía del Cono Sur, LI Congreso Argentino de Anatomía, XXXV Congreso Chileno de Anatomía, II Congreso Uruguayo de Anatomía, XX Congreso de 
Ciencias Morfológicas de Corrientes, II Congreso Argentino de Técnicas Anatómicas y VI Jornadas Argentinas de Anatomía para Estudiantes de las Ciencias de la Salud. En Corrientes, Argentina, durante los días 16, 17 y 18 de octubre de 2014. Presidente del XVI Congreso de Anatomía del Cono Sur: Prof. Dr. Arturo M. Gorodner (Argentina).

XVII Congreso de Anatomía del Cono Sur, XXXVI Congreso Chileno de Anatomía y XIII Simposio Ibero latinoamericano de Terminologia Anatomica, Histologica y Embryologica (SILAT XIII), en Valdivia, Chile, durante los días 11, 12 y 13 de noviembre de 2015. Presidente: Prof. María Eliana Sanz J. (Chile).

XVIII Congreso de Anatomía del Cono Sur, LIII Congreso Argentino de Anatomía, XXXVII Congreso Chileno de Anatomía, IV Congreso Argentino de Técnicas Anatómicas y VIII Jornadas Argentinas de Anatomía para Estudiantes de Ciencias de la Salud, en Buenos Aires, Argentina durante los días 25, 26 y 27 de agosto de 2016. Presidente del Congreso Chileno: Prof. Dr. Juan Carlos López Navarro (Chile) y Presidente de los Congresos Argentino y del Cono Sur: Prof. Dr. Marcelo Acuña (Argentina).

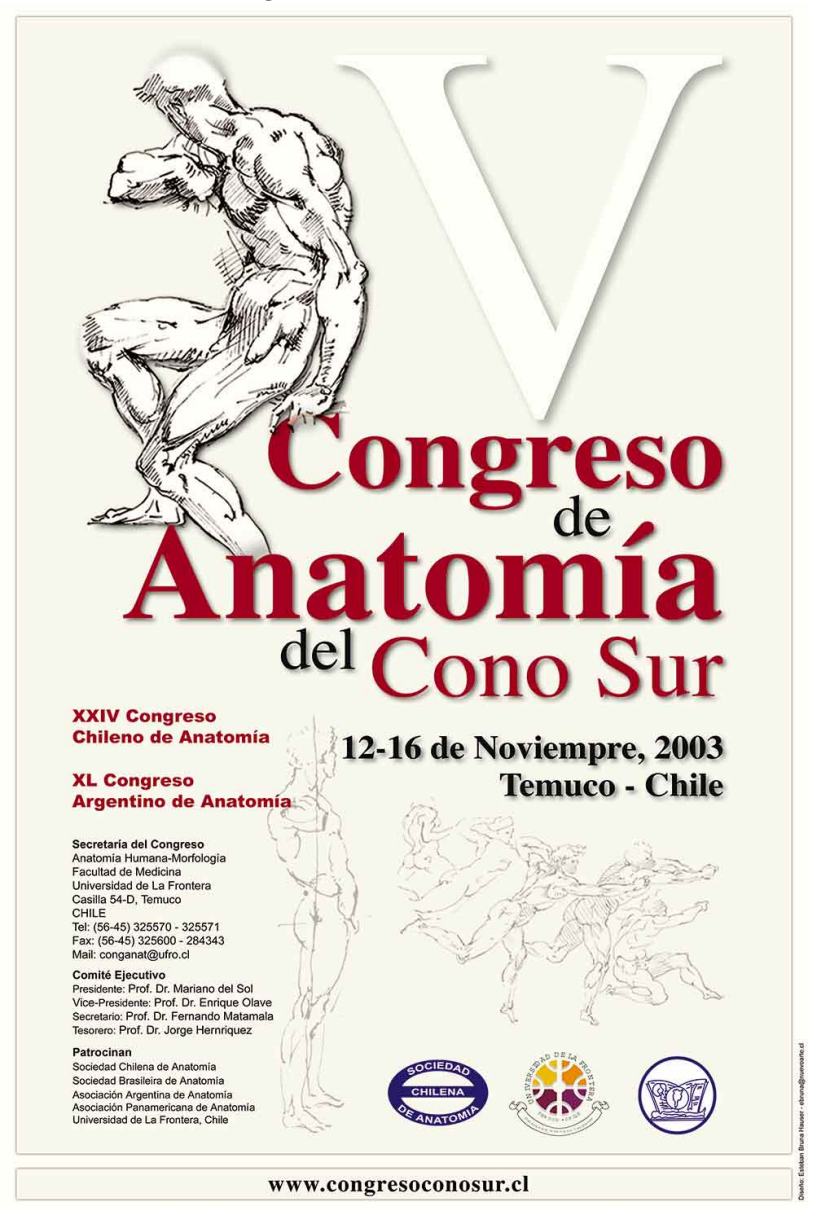

Fig. 4. Poster del V Congreso de Anatomía del Cono Sur.
XIX Congreso de Anatomía del Cono Sur, XXXVIII Congreso Chileno de Anatomía y III Olimpiadas de Anatomía de Estudiantes, en Concepción, Chile, durante los días 8, 9 y 10 de noviembre de 2017. Presidente: Prof. Rodolfo R. Sanzana Cuche (Chile).

XX Congreso de Anatomía del Cono Sur y XVI Simposio Ibero latinoamericano de Terminologia Anatomica, Histologica y Embryologica (SILAT XVI), XII Jornadas Chilenas de Anatomía y IV Encuentro Regional de Morfología, en Pucón, Chile, durante los días 4, 5 y 6 de octubre de 2018. Presidente del SILAT: Prof. Dr. Mariano del Sol (Chile) y Presidente del Congreso del Cono Sur: Prof. Dr. Nicolás Ernesto Ottone (Chile).

XXI Congreso de Anatomía del Cono Sur, XIX Congreso Panamericano de Anatomía, LVI Congreso Argentino de Anatomía; XL Congreso Chileno de Anatomía; I Congreso Ecuatoriano de Ciencias Morfológicas; XVII Simposio Ibero Latinoamericano de Terminologia Anatomica, Histologica y Embryologica (SILAT XVII), I Simposio Sudamericano de la International Society for Plastination (ISP) y XI Jornadas Argentinas de Anatomía para Estudiantes de Ciencias de la Salud, en Buenos Aires, Argentina, durante los días 27, 28, 29, 30 y 31 de mayo de 2019. Presidente del Congreso Panamericano: Prof. Dr. Rubén Daniel Algieri (Argentina), Presidente del Congreso del Cono Sur: Prof. Dr. Mariano del Sol (Chile), Presidente del Congreso Argentino: Prof. Dr. Gustavo Grgicevic (Argentina), Presidente del Congreso Chileno: Prof. Dr. Reinaldo Soto (Chile) y Presidente del Congreso Ecuatoriano: Prof. Dr. Marco Guerrero (Ecuador).

\section{ALGUNOS DETALLES DE LOS CONGRESOS V, X, XV y XX.}

V Congreso de Anatomía del Cono Sur (Temuco, 2003).

Fue un congreso muy masivo y participaron morfólogos de América Latina, Europa, Asia y África. (Fig. 4).

Entre los conferencistas podemos destacar entre otros a los profesores doctores de los siguientes países: a) España: María José Blánquez, de la Universidad Complutense de Madrid; y Javier Regadera González, de la Universidad Autónoma de Madrid; b) Israel: Baruch Arensburg, de la Universidad de Tel Aviv; y c) Japón: Kan Kobayashi, de Nipón Dental University.

Del Cono Sur estuvieron presentes profesores doctores de: a) Brasil: Enrique Ayres Vasconcellos y Carlos Alberto Mandarim de-Lacerda, ambos de la Universidade do Estado do Rio de Janeiro; Nadir Eunice Valverde Barbato de Prates, de la Universidade de São Paulo; Márcia Barbosa-Águila, de 


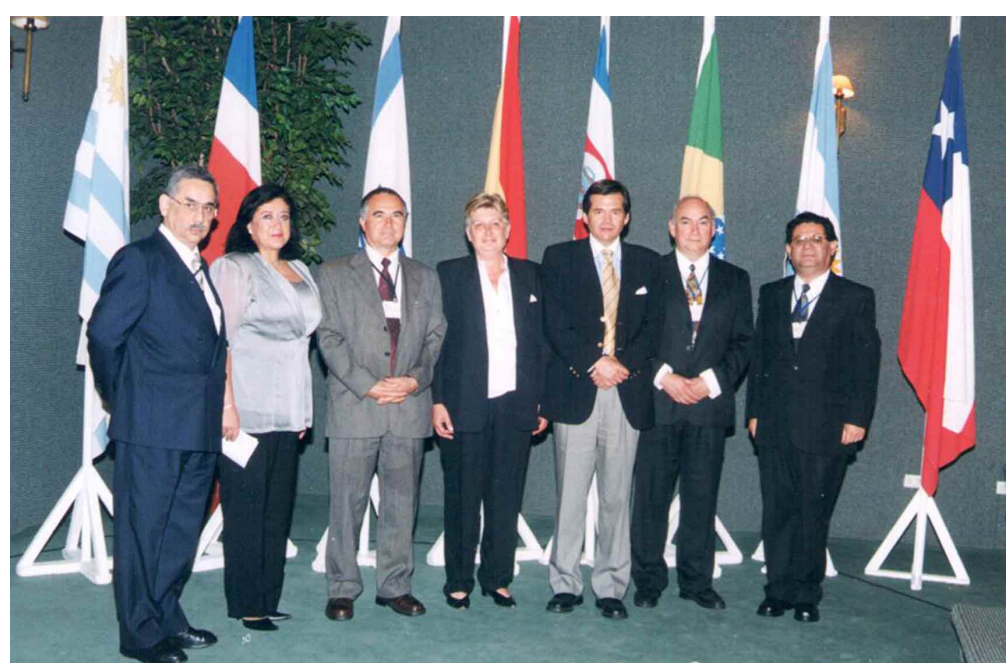

Fig. 5. Autoridades y Miembros del Comité Organizador del V Congreso de Anatomía del Cono Sur. De izquierda a derecha se encuentran Prof. Dr. Wilfried Diener Ojeda, Decano Facultad de Medicina, Universidad de La Frontera-Chile; Prof. Dra. Nadir Eunice Barbato Valverde de Prates, Presidente de la Sociedade Brasileira de Anatomia; Prof. Dr. Hermes Bravo Contreras, Presidente de la Sociedad Chilena de Anatomía; Prof. Sergio Bravo Escobar, Rector de la Universidad de La Frontera-Chile; Prof. Dr. Mariano del Sol Calderón, y Prof. Fernando Matamala Vargas, Presidente y Secretario del Comité Organizador, respectivamente.

UniRio, Rio de Janeiro; Octavio Binvignat-Gutiérrez de la Faculdade da Serra Gaúcha; Benedicto de Campos-Vidal, de la Universidade de Campinas; José Carlos Prates, de la Universidade Federal de São Paulo - Escola Paulista de Medicina; Célio de Sousa-Rodrigues, de la Universidade Federal de Alagoas, Maceio. b) Argentina: Adrián Barceló, de la Facultad de Medicina, H. A. Barceló, Buenos Aires; AliciaCostamagna, de la Universidad Nacional del Litoral; Ricardo Jorge Losardo, de la Universidad de Buenos Aires. c) Chile: Hermes Bravo C. y Oscar Inzunza H., de la Pontificia Universidad Católica de Chile, Santiago; Eduar-

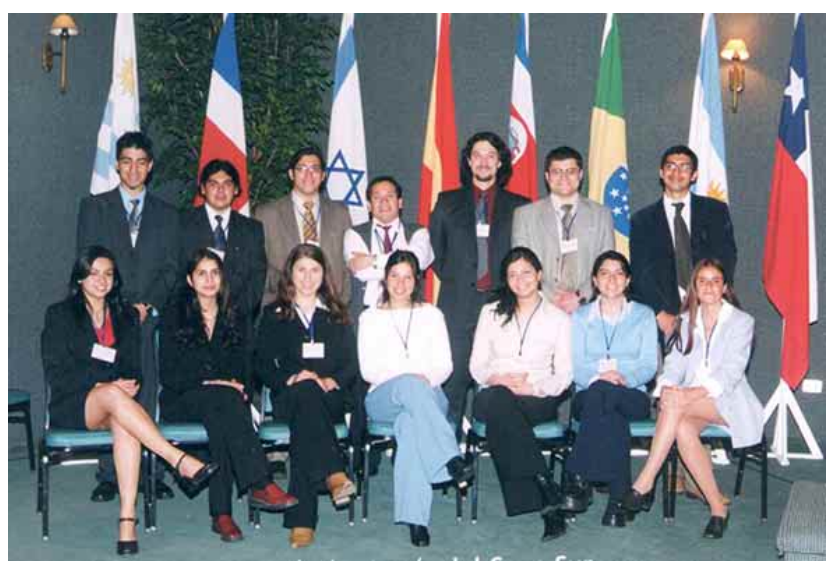

Fig. 6. Prof. Héctor Silva Mella (al centro), Director de Infraestructura del V Congreso de Anatomía del Cono Sur, junto estudiantes de la Facultad de Medicina de la Universidad de La Frontera quienes apoyaron la parte de secretaría e infraestructura. do Bustos-Obregón y Mariana Rojas-Rauco de la Universidad de Chile; Francisco Nualart, de la Universidad de Concepción; Jorge Henríquez-Pino, Fernando Matamala-Vargas y Luis Salazar, de la Universidad de La Frontera, Temuco; entre otros.

Se destacaron un curso de 6 horas de duración sobre investigación empleando estereología y morfología cuantitativa sin distorsión, a cargo del Prof. Dr. Carlos Alberto Mandarim-de-Lacerda y una mesa redonda que trató sobre la Integración de las Ciencias Morfológicas en las Universidades, cuyo coordinador fue el Prof. Dr. Alberto Rodríguez Torres. Ambas tuvieron una numerosa concurrencia y despertó un gran interés en el auditorio.

Las delegaciones más numerosas fueron de argentinos, brasileños, chilenos y uruguayos, pero también estuvieron presentes profesores e investigadores de otros países de América, Europa, Asia y África. Fue muy bien recibida la asistencia de una delegación de Rumania, con la presencia de los Profs. Dres. Petru Bordei, Petru Matusz y Delia Elena Zahoi; en su carácter de Presidente, Secretario General y Secretaria de la Sociedad Rumana de Anatomistas, respectivamente.

El Congreso se realizó en el Hotel Terraverde, situado en las faldas del Monumento Natural el Cerro Ñielol de Temuco: un parque y reserva ecológica de la ciudad de Temuco. El hotel de 5 estrellas a pesar de su gran capacidad, fue colmado por la masiva asistencia y muchos congresistas tuvieron que alojarse en otros nueve hoteles y en un aparthotel de la ciudad. Un elegante hotel, que poco tiempo después fue vendido y destinado al Centro de Justicia de Temuco. Fotografías en el día de la inauguración (Fig. 5) y durante el congreso (Figs. 6 y 7 ).

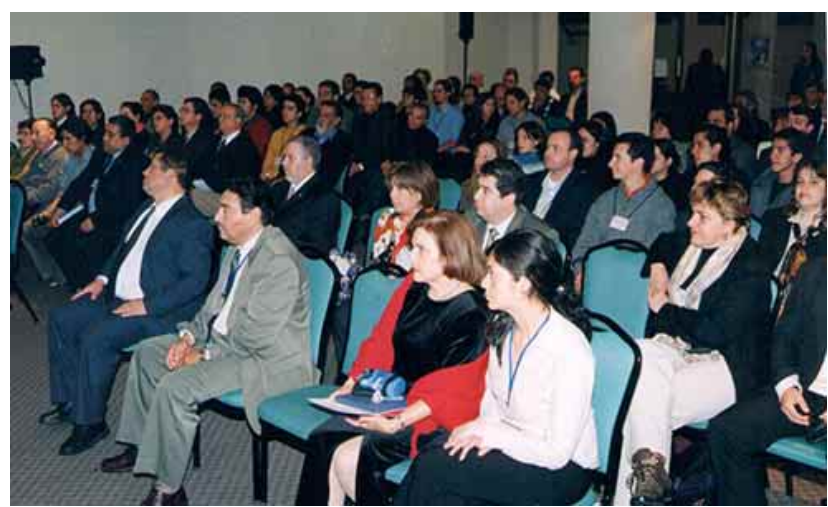

Fig. 7. Asistentes en uno de los salones del V Congreso de Anatomía del Cono Sur, 2004. 
El hotel Terraverde estuvo destinado exclusivamente para el desarrollo del Congreso. De este modo se protegió la privacidad del congreso y la seguridad de los congresistas. Se nos otorgó trato preferencial, habitaciones, personal administrativo y toda la hotelería estuvo a disposición. Se contó con un salón plenario para mil personas, además de otros tres salones para 200 a 300 personas y también un salón VIP. El espacio para la secretaría del congreso era importante y resultaba muy cómodo para la consulta de los congresistas.

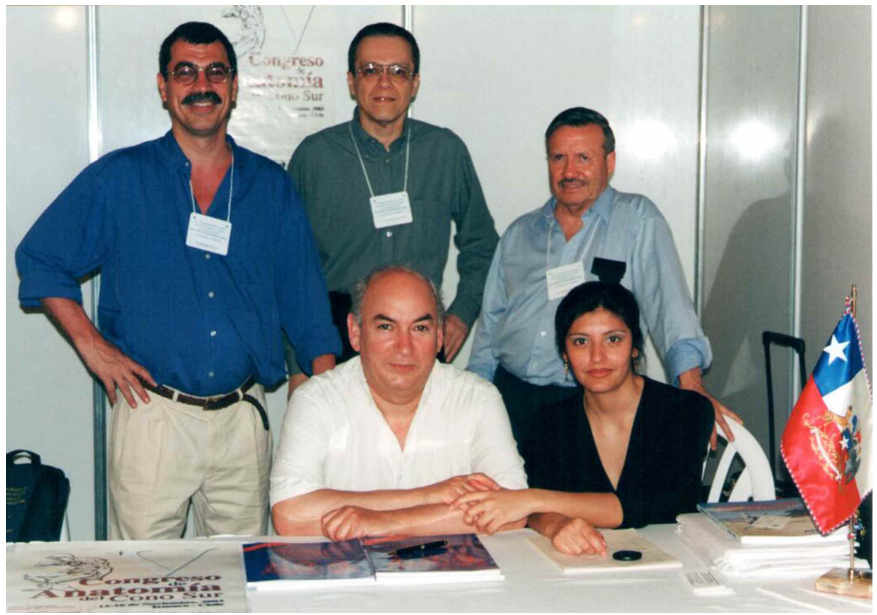

Fig. 8. En el stand de la revista International Journal of Morphology durante el V Congreso de Anatomía del Cono Sur. Se observan de pie de izquierda a derecha los Profs. Drs. Ricardo Losardo (Argentina), Carlos Alberto Mandarim de-Lacerda (Brasil) y Dr. Eduardo Mandiola Lagunas (Chile) y sentados los Prof Drs. Mariano del Sol y Bélgica Vásquez).

Coffee Breaks y cócteles no faltaron mezclándose los "piscos sours" peruanos y chilenos, almuerzos compartidos con chef de primer nivel y una cena buffet final bailable -con bar abierto- todo incluido en la inscripción. Por otra parte, durante el evento se llevaron a cabo numerosas actividades sociales y de camaradería con folclore chileno y latinoamericano a cargo de un excelente grupo de baile universitario. En el segundo y tercer día, la música estuvo presente en plenitud, una estudiantina y una tuna universitaria, antigua tradición que se originó en España y se extendió a esta parte del mundo, en especial al Cono Sur de América, animaron a los congresistas.

También los congresistas visitaron la Facultad de Medicina de la Universidad de La Frontera y fueron recibidos por las autoridades con comida típica chilena acompañado por supuesto de un gran vino chileno Cabernet Sauvignon. El último día, un paseo a la zona lacustre del sur de Chile, luego a las termas a recibir un buen baño para terminar con un gran churrasco o asado a la chilena que dieron por terminado el congreso. Como producto de todas las actividades científicas y sociales no faltaron los amores internacionales que terminaron unos meses después con un casamiento, entre una hermo- sa chilena y un apuesto morfólogo colombiano. Este congreso aún hoy es recordado por los asistentes; y tal vez sea considerado el mejor Congreso de Anatomía del Cono Sur realizado a la fecha. En la figura 8 se observan algunos profesores participantes del congreso en el stand de la revista International Journal of Morphology continuación de la Revista Chilena de Anatomóa y en su primer año de circulación.

\section{Congreso de Anatomía del Cono Sur (Belém, 2008)}

El 22 de noviembre de 2007, la Prof. Dra. Nadir Barbato de Prates (Ex Presidenta de la SBA), le envió un email al Dr. Mariano del Sol informándole que estaban haciendo las gestiones, a través de la Sociedade Brasileira de Anatomia ( $S B A$ ), para anexar el próximo Congreso Chileno y del Cono Sur de Anatomía al XXIII Congresso Brasileiro de Anatomia, que se realizaría en la ciudad de Belêm (Fig. 9). Este evento se haría por primera vez en la Amazonia, promoviendo la importancia de la Morfología en el currículum universitario. Uno de los que consolidó esta unión entre estos países fue también el Prof. Dr. Richard Halti Cabral, quien ocupaba la presidencia de la $S B A$ y se mantuvo en permanente contacto con el Dr. Mariano del Sol. Era el cuarto Congreso de Anatomía del Cono Sur que se realizaba en Brasil.

Participaron importantes académicos e investigadores del área de la anatomía y de la morfología, destacándose la presencia de los Profs. Dres. Keith L. Moore, de la Universidad de Toronto - Canadá (autor del libro "Anatomía Orientada para Clínica" y miembro fundador y Ex Presidente de la American Association of Clinical Anatomists) y Udo Schumacher, del Centro Médico Universitario de Hamburgo-Eppendorf, Alemania (autor del "Prometheus: Atlas de Anatomía") quienes impartieron conferencias magistrales inolvidables.

Terminado el Congreso, algunos profesores extranjeros, previamente vacunados contra la fiebre amarilla, comenzaron su "turismo académico" navegando

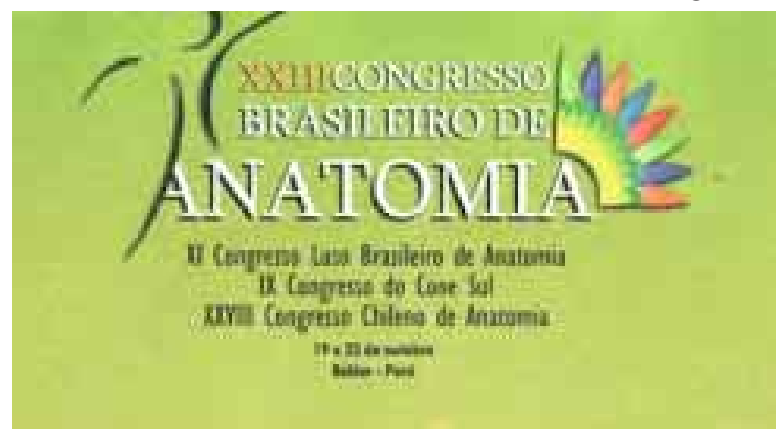

Fig. 9. Certificado del X Congreso de Anatomía del Cono Sur realizado en Belêm, Brasil. 
por el Amazonas con destino a la isla de Marajó. El baño en el río más ancho del mundo, el paseo en búfalo y la estancia en un complejo turístico en la isla hicieron de la travesía un recuerdo inolvidable. Las anécdotas de dicho viaje darían para varios "papers". (Fig. 10).

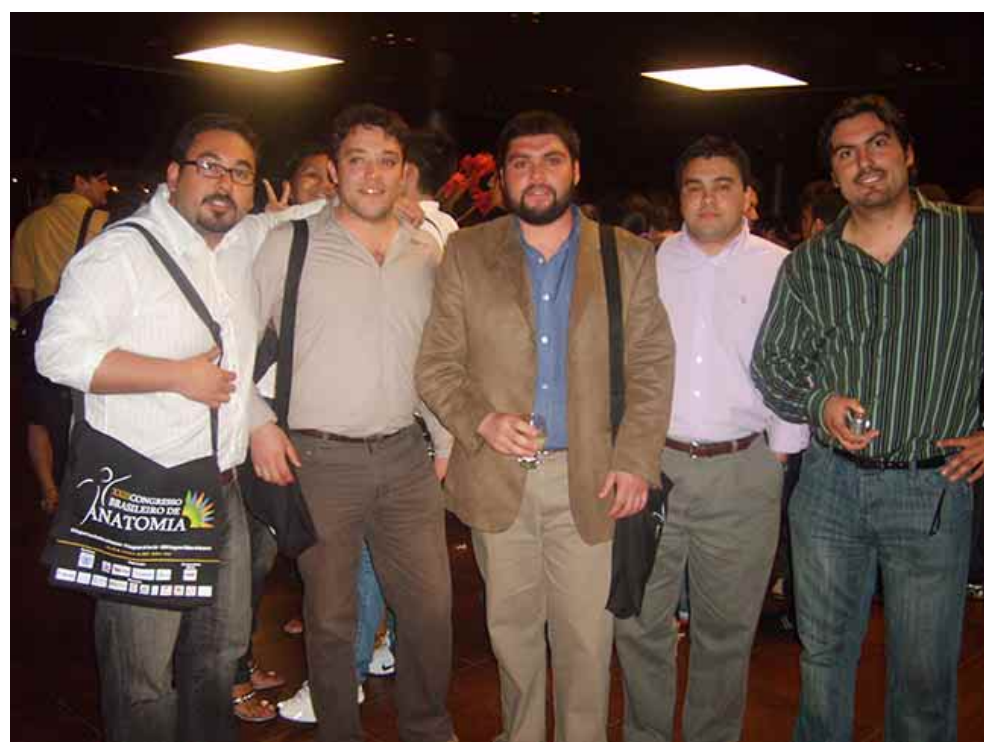

Fig. 10. Participantes chilenos del X Congreso de Anatomía del Cono Sur en Belêm, Brasil. De izquierda a derecha Dres. Ignacio Roa, Mario Cantín, César Coronado, Juan Pablo Pacheco y Guillermo Salgado.

\section{Congreso de Anatomía del Cono Sur (Talca, 2013)}

El Acto de Inauguración del Congreso se realizó el 20 de noviembre en el teatro Abate Molina, localizado en la Casa Central de la Universidad de Talca y estuvo a cargo del Prof. Dr. Carlos Alberto Mandarim-de-Lacerda de la Universidade do Estado do Rio de Janeiro, Brasil. (https://www.youtube.com/watch?v=Bbgx_IcTVrM) (Fig. 11)

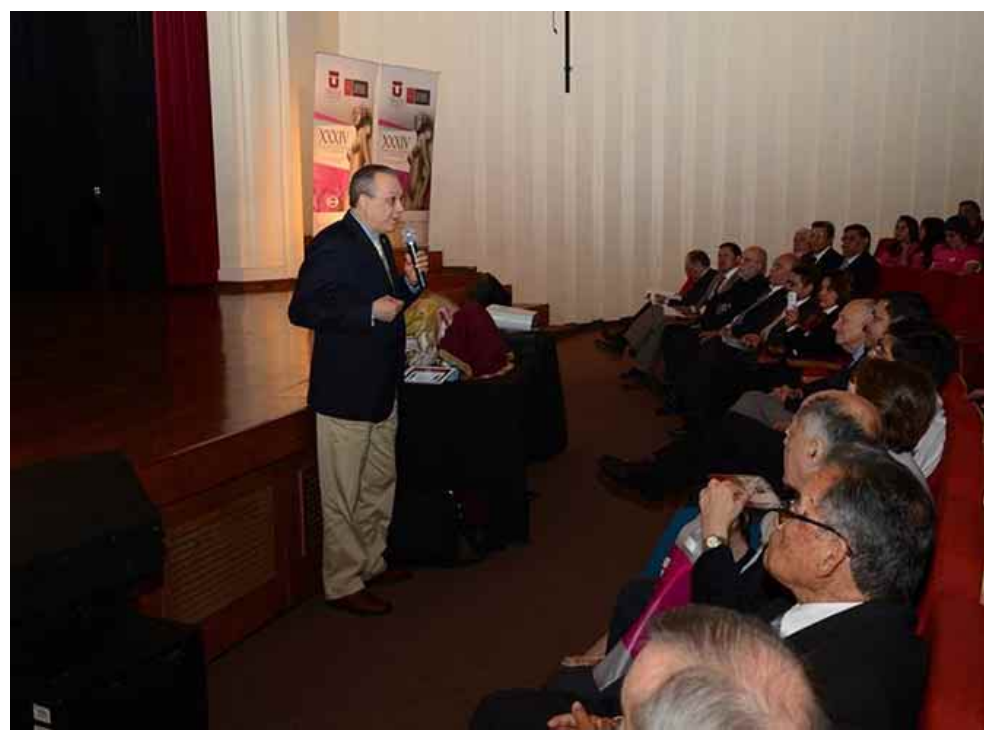

Fig. 11. Prof. Dr. Carlos Alberto Mandarim-de-Lacerda dictando la Conferencia Inaugural del XV Congreso de Anatomía del Cono Sur (Talca, 2013).
El programa científico fue realizado en el Hotel Casino de la ciudad, contó con una amplia participación de morfólogos de América, en especial argentinos, brasileños y chilenos. Dictaron conferencias, entre otros, los Profs. Dres.: Eduardo Bustos-Obregón, Omar Fuentes Díaz, Pablo Lizana Arce y Mauro Parra, de Chile; Marcia Barbosa y Carlos Mandarim-de-Lacerda, de Brasil; Eduardo Pró, de Argentina; Santiago Aja Guardiola y Manuel Arteaga, de México y Ricardo Jiménez-Mejías, de Colombia; entre otros. Tres mesas redondas (Educación y Anatomía; Publicación, Investigación y Terminología en Anatomía; e Historia de la Morfología) y tres mini cursos (Técnicas Anatómicas, Embriología Humana y Suturas) completaban el programa y despertaron gran interés y participación.

En la parte social y cultural no podía faltar la visita a una viña, ya que es una de las regiones vitivinícolas más importantes de Chile, con una fuerte tradición del cultivo de la vid y en la elaboración de excelentes vinos. La región vinícola del Valle del Maule queda a unos 260 kilómetros al sur de Santiago y es una de las dos regiones, que reclama el título de "Cuna del vino chileno" ya que se remonta a los tiempos de los conquistadores españoles, que en el siglo XVI ya la cultivaban. En honor a esta tradición, se realizó un Curso de "Cata de vinos" en la ciudad de Quinamávida, y no faltaron estudiantes de este curso en reprobar "el examen de degustación" debido a las condiciones en que quedaron después de la cata.

\section{Congreso de Anatomía del Cono Sur (Pucón, 2018)}

Este Congreso de Anatomía del Cono Sur se realizó por primera y la única vez de manera separada de los Congresos Argentino, Brasileño y Chileno y Panamericano de Anatomía. Sin embargo, se unió al SILAT, el cual depende de la Asociación Panamericana de Anatomía y de la Academia Panamericana de Anatomía y fue su versión XVI. Este SILAT fue de gran relevancia histórica, ya que se realizó en homenaje a la reciente y triste partida del Prof. Dr. Rolando Cruz Gutiérrez. Fig. 12.

El Presidente de este Congreso del Cono Sur, fue el Dr. Nicolás Ottone, argentino, miembro de la Asociación Argentina de Anatomía; y 


\section{Congreso de Anatomía} del Cono Sur

XVI Simposio Iberolatinoamericano de Terminologia Anatomica, Histologica, Embryologica. SILAT en homenaje al

Prof. Dr. Rolando Cruz Gutiérrez

XII Jornadas Chilenas de Anatomía

IV Encuentro Regional de Morfología

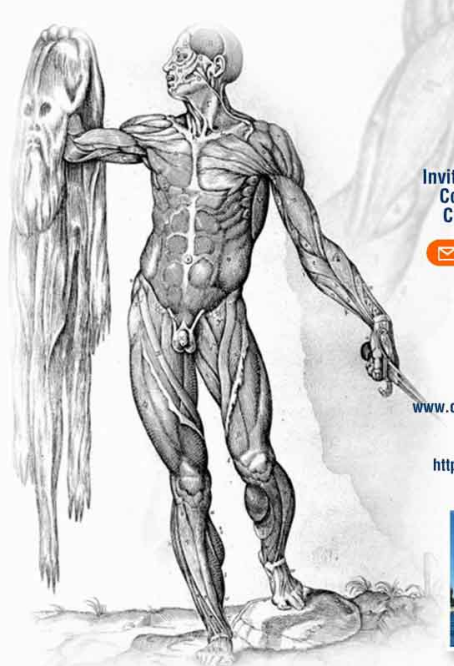

4,5 y 6 de Octubre de 2018 Pucón, Chile. Gran Hotel Pucón vitados Nacionales y Extranjeros Conferencias, Mesas Redondas,
Cursos, Temas Libres y Paneles $\square$ Fecha limite de envio de trabajos: 30 de Jullio de 2018 - 23:59 h (1) Consultas: nicolas.ottone@ufrontera.c
sandra.villa@ufrontera.cl Gr Sitio Web: (f) Facebook: AnatoConoSurXVISILAT2018

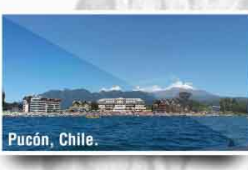

OR G A I Z A A U S PICIOS:
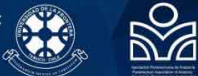

Fig. 12. Poster del XX Congreso de Anatomía del Cono Sur realizado el 2018 en Pucón, Chile. recién radicado en Chile, que había ingresado a la Sociedad Chilena de Anatomía, siendo actualmente uno de sus directores; y además Profesor Asociado de la Facultad de Odontología de la Universidad de La Frontera, Chile. Este es uno de los ejemplos de esta unión científica trasandina. (Fig. 13).

El congreso logró reunir a destacados morfólogos de América Latina, entre los cuales podemos destacar a los Profs. Dres. Nadir Barbato de Prates, Valeria Fazán, Telma Masuko, Eulampio Netto, y José Carlos Prates, de Brasil; Julio Cárdenas-Valenzuela, Carlos Manterola-Delgado, Francisco Nualart, Nicolás Ottone y Raúl Sánchez, de Chile; Rubén Daniel Algieri, Rodolfo Ávila, Homero Bianchi, Carlos Blanco, Ricardo Losardo y María Elena Samar, de Argentina; Gustavo Armand-Ugon y Alejandro Russo, de Uruguay; Jorge Duque Parra, de Colombia; y Carlos Baptista, de Estados Unidos de Norteamérica. Todos ellos participaron activamente en el Congreso dictando conferencias y/o dirigiendo mesas redondas.

La Conferencia Inaugural estuvo a cargo del Prof. Dr. Alberto Rodríguez Torres, Maestro de la Anatomía de Chile, quien dictó la conferencia «Los albores de la Sociedad Chilena de Anatomía» (Fig. 14). El Acto de Clausura fue coronado por una brillante conferencia dictada por el Prof, Dr. Carlos Augusto Camargo Baptista de la Universidad de Toledo, Ohio, Estados Unidos titulada «The art and science of plastination»(Fig. 15).

El congreso terminó con un paseo turístico-cultural, visitando el lago Caburgua y con un almuerzo de confraternidad en una de las típicas termas-balneario del sector montañoso de la región. Las Figuras 16-20 muestran la participación de los congresistas.

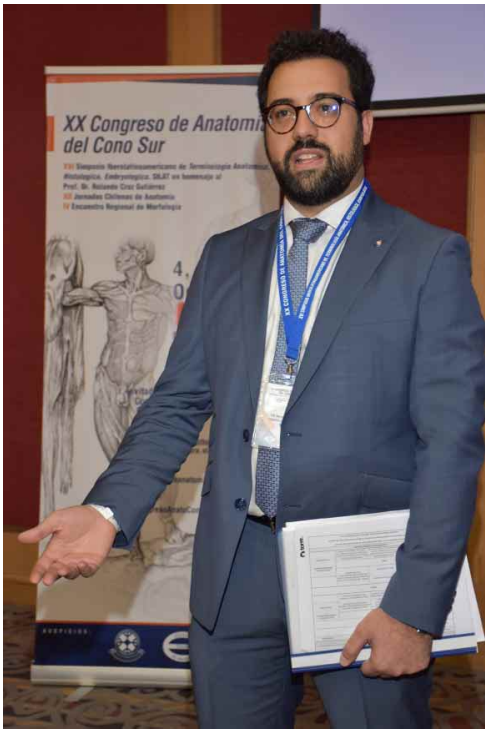

Fig. 13. Prof. Dr. Nicolás E. Ottone, Presidente del XX Congreso de Anatomía del Cono Sur, Pucón 2018.

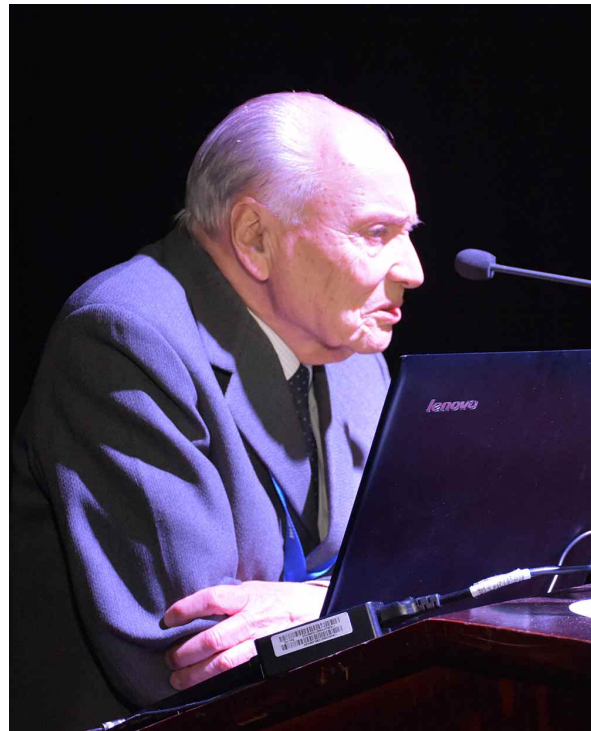

Fig. 14. Prof. Dr. Alberto Rodríguez Torres dictando la conferencia inaugural en el XX Congreso de Anatomía del Cono Sur, Pucón, 2018).

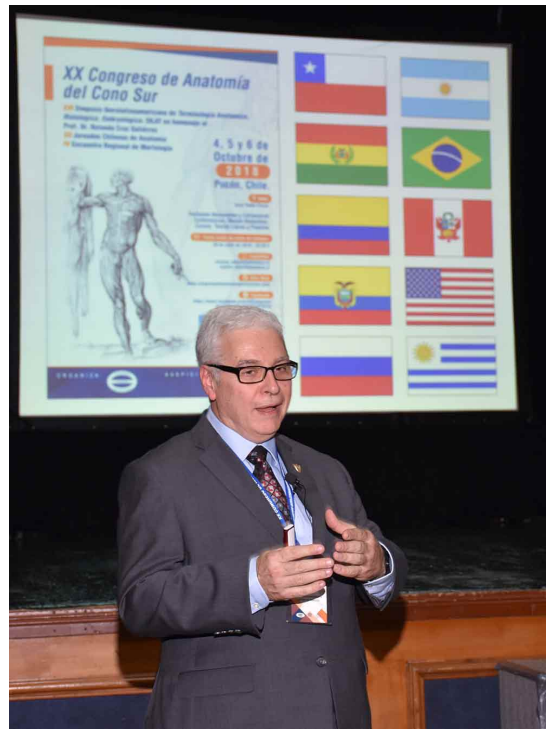

Fig. 15. Prof. Dr. Carlos Baptista durante el cierre del XX Congreso de Anatomía del Cono Sur, Pucón, 2018. 

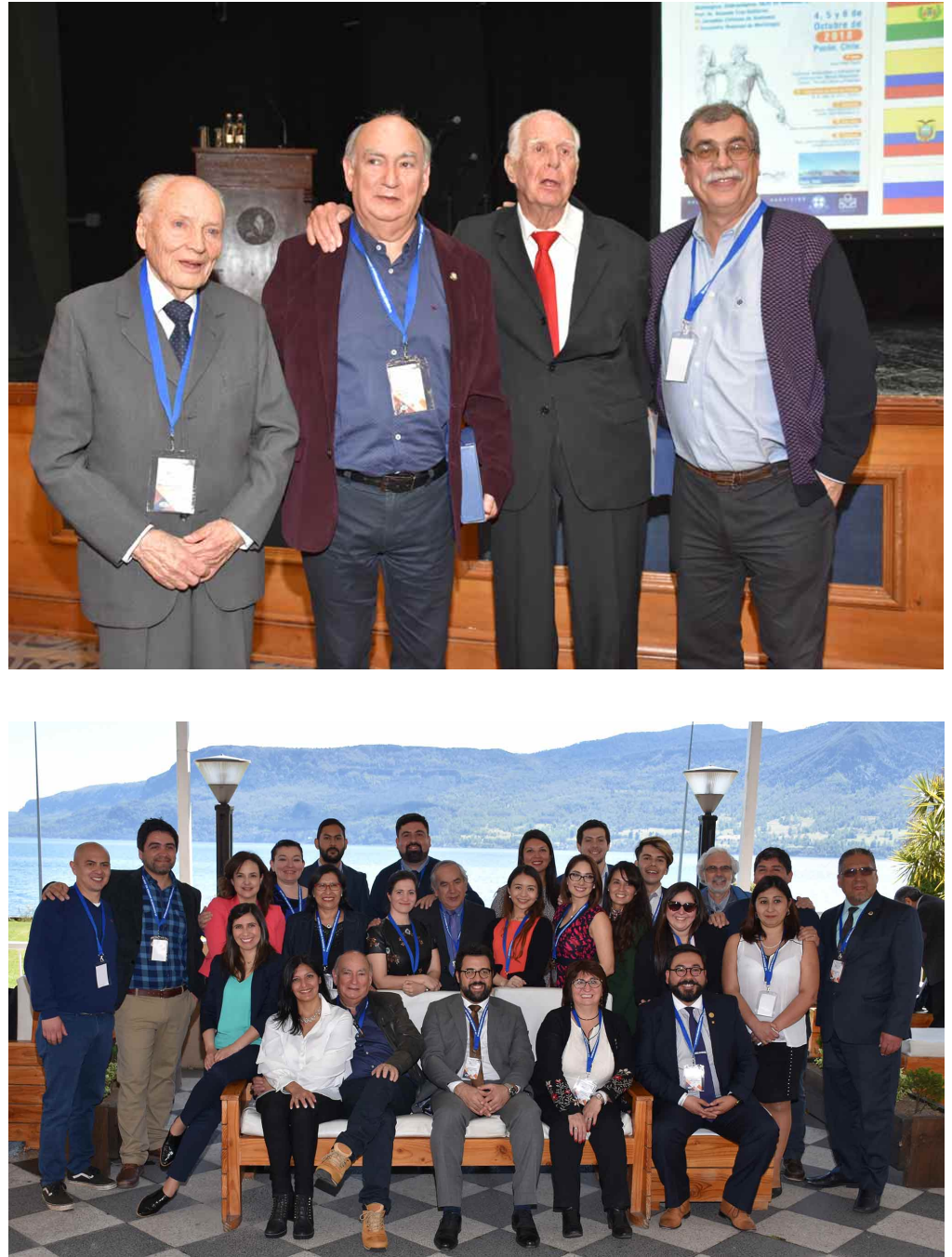

Fig. 17. Foto grupal de estudiantes y profesores del Programa de Doctorado en Ciencias Morfológicas de la Universidad de La Frontera asistentes al XX Congreso de Anatomía del Cono Sur realizado en octubre de 2018 en Pucón, Chile.

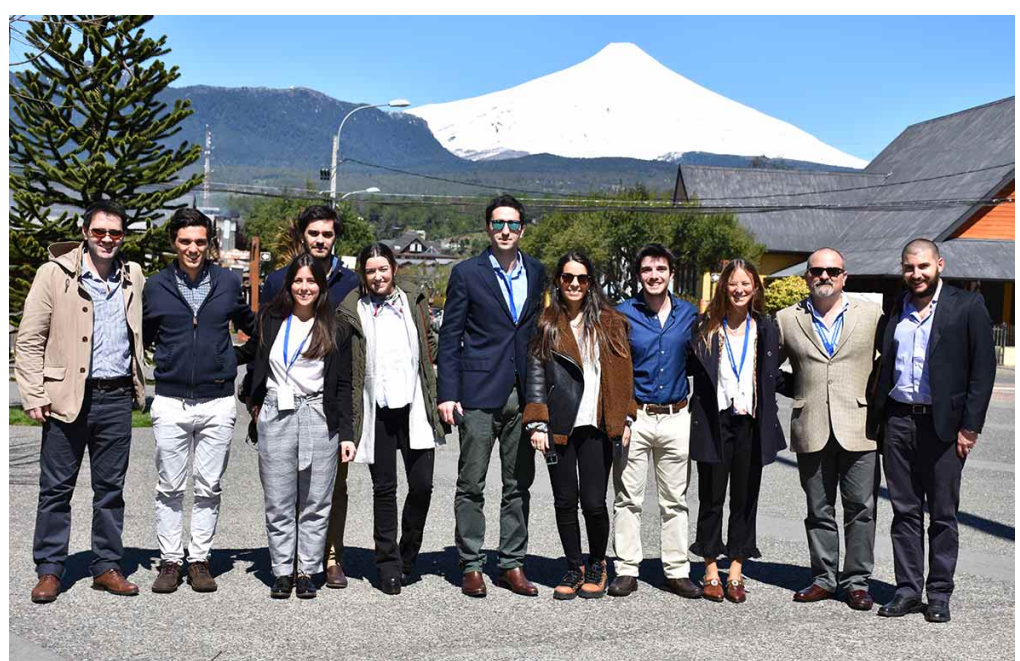

Fig. 18. Participantes de la delegación uruguaya en el XX Congreso de Anatomía del Cono Sur realizado en octubre de 2018 en Pucón, Chile.
Fig. 16. Ex Presidentes de la Asociación Panamericana de Anatomía asistentes al XX Congreso de Anatomía del Cono Sur realizado en el mes de octubre de 2020 en la ciudad de Pucón, Chile. De izquierda a derecha se encuentran los Profs. Dres. Alberto Rodríguez Torres y Mariano del Sol Calderón ambos Maestros de Anatomía de Chile; Prof. Dr. José Carlos Prates de la Escola Paulista de Medicina - Universidade Federal de São Paulo, Brasil y el Prof. Dr. Ricardo Jorge Losardo de la Universidad del Salvador (USAL), Buenos Aires, Argentina.

\section{Congresos Argentinos de Anatomía}

Por distintas razones algunos Congresos Argentinos no se pudieron realizar en forma conjunta con los Congresos del Cono Sur. Sin embargo, los resúmenes de las versiones 42 y 43 fueron publicados en la revista International Journal of Morphology (Asociación Argentina de Anatomía, 2006; Asociación Argentina de Anatomía, 2007).

De esta manera, los siguientes Congresos Argentinos de Anatomía se realizaron en forma separada: XLII, noviembre de 2005 (ciudad de Mar del Plata); XLIII, octubre de 2006 (ciudad de Rosario); XLIV, noviembre de 2007 (ciudad de La Plata); XLV, octubre de 2008 (ciudad de Mendoza); XLVI, septiembre de 2009 (ciudad de Corrientes); XLVII, octubre de 2010 (ciudad de Cipolletti); XLVIII, octubre de 2011 (ciudad de Santo Tomé); XLIX, septiembre de 2012 (ciudad de Buenos Aires); L, octubre de 2013 (ciudad de Rosario); LII, septiembre de 2015 (ciudad de La Rioja); LIV, septiembre de 2017 (ciudad de La Plata); y LV, junio de 2018 (ciudad de Mendoza).

\section{Congresos Chilenos de Anatomía}

Como fue mencionado anteriormente, solamente el año 2018 el XXXIX Congreso Chileno de Anatomía no se realizó junto al Congreso de Anatomía del Cono Sur, ya que en julio de ese año, se unió al XXVIII Congresso Brasileiro de Anatomia evento realizado en la ciudad de João Pessoa, Brasil. 


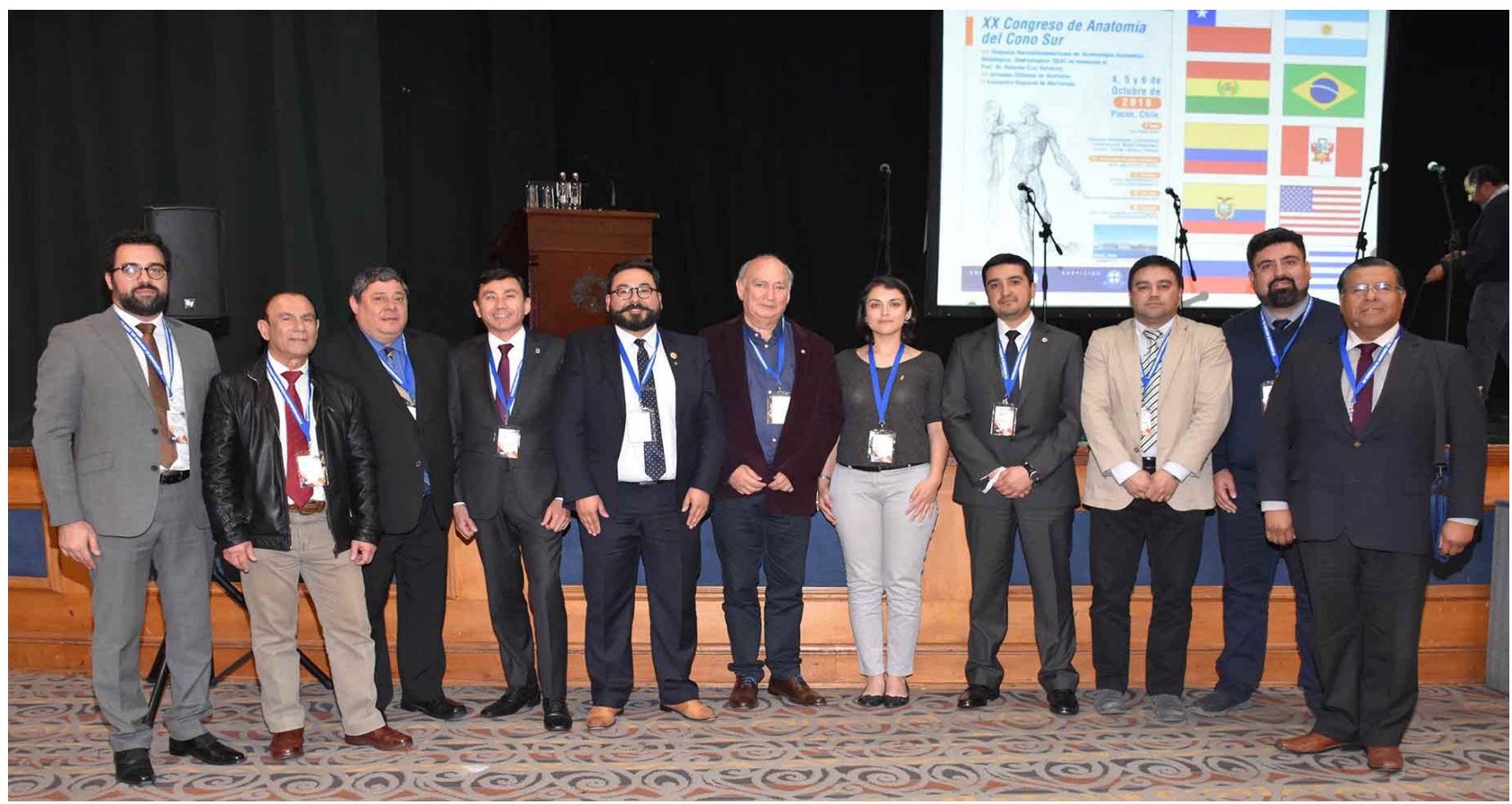

Fig. 19. Miembros de la Directiva de la Sociedad Chilena de Anatomía asistentes al XX Congreso de Anatomía del Cono Sur. De izquierda a derecha los Profs. Dres. Nicolás E. Ottone Cappiello (Universidad de La Frontera), Omar Espinoza Navarro (Universidad de Tarapacá), Reinaldo Soto Norambuena (Universidad de Los Andes), Mauricio Villarroel Guerra (Universidad de Playa Ancha), Ignacio Roa Henríquez (Universidad de Talca), Mariano del Sol Calderón (Universidad de La Frontera), Viviana Toro Ibacache (Universidad de Chile), Pablo Lizana Arce (Pontificia Universidad Católica de Valparaíso), Juan Pablo Pacheco Muñoz (Universidad San Sebastián), Andrés Riveros Valdés (Universidad de Concepción y Juan Carlos López Navarro (Universidad de Los Andes).

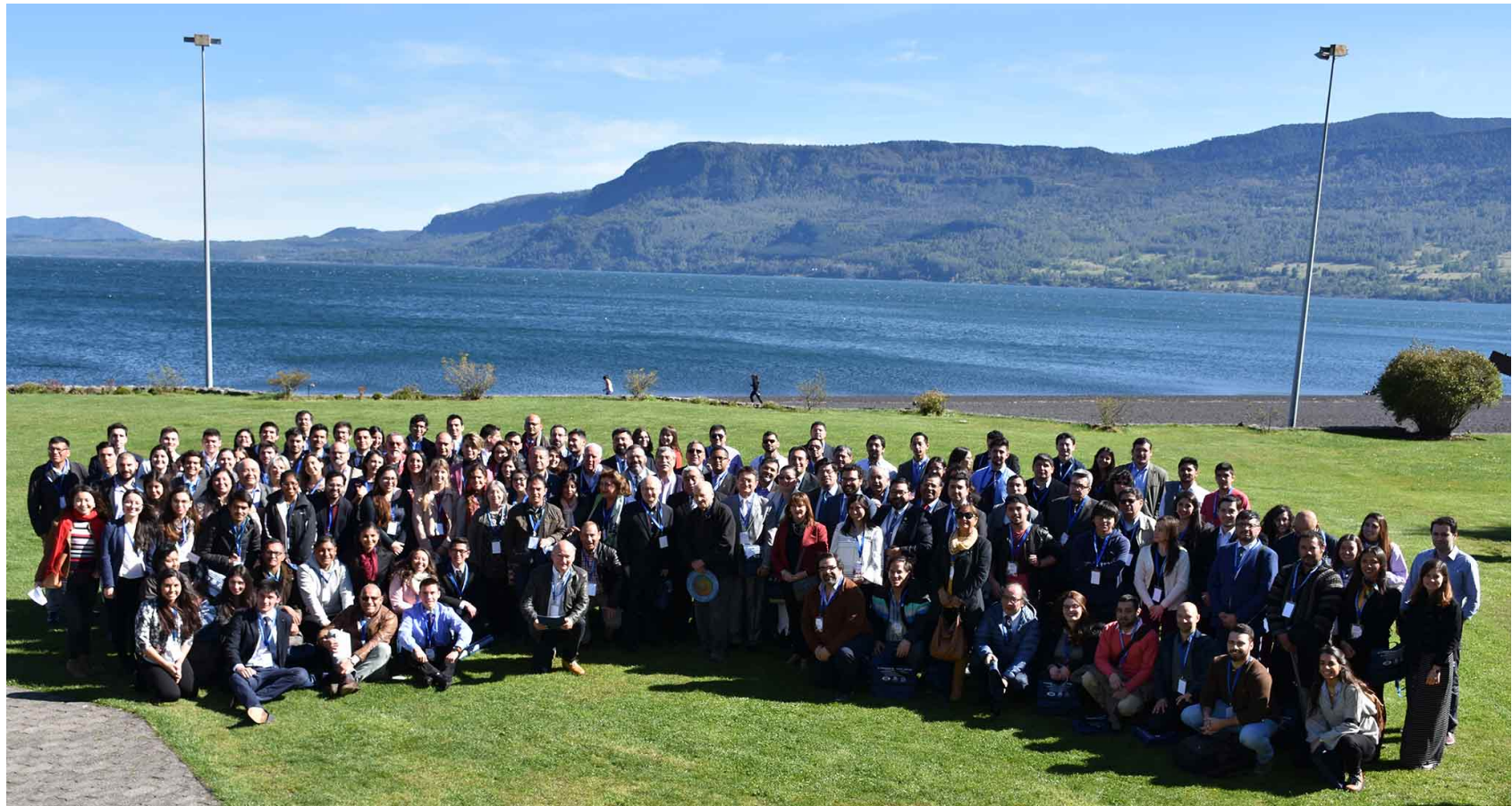

Fig. 20. Foto grupal de algunos de los asistentes al XX Congreso de Anatomía del Cono Sur realizado en octubre de 2018 en Pucón, Chile. Fotografía tomada en los jardínes del Hotel, frente al lago Villarrica. Se observan profesores y estudiantes de postgrado de Argentina, Bolivia, Brasil, Chile, Colombia, Ecuador, Estados Unidos, Rusia y Uruguay. 


\section{COMENTARIOS}

La necesidad de estimular el desarrollo y el mejoramiento de la docencia y la investigación de la Anatomía y de las Ciencias Morfológicas en general, en los distintos países, fue un motor común en la década del sesenta que no tuvo fronteras en América. La región del Cono Sur de América no quedó exceptuada de este fenómeno y con ese fin se crearon distintas sociedades anatómicas.

Así, en 1952, se fundó la Sociedad Brasileña de Anatomía. Su mentor fue Renato Locchi, discípulo de Alfonso Bovero (ítalo-brasileño) (Talamoni \& Bertolli, 2014).

Mientras, en 1964, en el Río de La Plata, se creó una sociedad de anatomía que reunía a dos países del continente americano, Argentina y Uruguay, y se denominó Sociedad Rioplatense de Anatomía, cuyos mentores fueron José Luis Martínez (Argentina) y Alfredo Ruiz-Liard (Uruguay). Años más tarde, se llamaría Asociación Rioplatense de Anatomía. Finalmente, daría lugar a la creación de la Asociación Argentina de Anatomía y de la Sociedad Uruguaya de Anatomía, representando cada una a sendos países.

Es de destacar que la primera sociedad de anatomía que reunió a varios países en el continente americano fue la Asociación Centroamericana de Anatomía, creada en 1965. Sus mentores fueron Manuel Sigarán-Ramírez (El Salvador), José Ramiro Rivera (Guatemala) y Rolando CruzGutiérrez (Costa Rica). Años más tarde, se llamaría Asociación Centroamericana y del Caribe de Anatomía (SigaránRamírez et al., 2018).

En 1966, se fundó la Asociación Panamericana de Anatomía, cuyo mentor fue Liberato DiDio (Brasil), logrando la expresión máxima de integración continental (Losardo, 2009). Es de resaltar que la realización de los sucesivos congresos panamericanos en esta región en 1978, 1981, 1984 y 1987 en San Pablo, Buenos Aires, Punta del Este (Uruguay) y Santiago de Chile, respectivamente, lograron acercar -por primera vez- a los anatomistas de estos cuatro países vecinos. Esta situación, a nuestro criterio, fue el germen de la unión y confraternidad de estos cuatro países limítrofes.

En 1979, se creó la Sociedad Chilena de Anatomía, cuyos mentores fueron Abraham Wainstein, Alberto Rodríguez-Torres, Humberto Güiraldes del Canto y Hugo Hernández-Parada (Rodríguez \& Cárdenas, 2017; Cárdenas \& Rodríguez, 2018).

En cuanto al perfeccionamiento personal que buscaban los anatomistas de estas regiones de América del Sur debemos resaltar que, en la primera mitad del siglo XX, se realizaban esfuerzos individuales con el fin de buscar formación académica en Europa, principalmente en Francia, Italia y Alemania. Pero a partir de la década del 80, gracias a la relación profesional entre Alberto Rodríguez-Torres (Chile) y José Carlos Prates (Brasil) se concretaron pasantías o rotaciones de profesores chilenos en la Escuela Paulista de Medicina (USP), que funcionó como un centro formador de morfólogos de primer nivel, bajo la tutoría del mismo Prates (Cárdenas \& Rodríguez). Recordemos que la Escuela Paulista de Medicina poseía un centro anatómico de excelencia, gracias al esfuerzo inicial de Alfonso Bovero y continuado por Renato Locchi (Talamoni \& Bertolli). Con los años, un grupo de anatomistas chilenos que se perfeccionaron en aquel centro, deciden replicar en la Universidad de La Frontera, en Temuco, aquel exitoso emprendimiento con el fin de facilitar a los profesores de Hispanoamérica su formación académica en las ciencias morfológicas. Así se creó el Magíster en Ciencias, Mención Anatomía en el año 2003; y posteriormente, en el año 2010, el Programa de Doctorado en Ciencias Morfológicas, el cual ha recibido a la fecha estudiantes de Argentina, Brasil, Bolivia, Chile y Ecuador. Estas escuelas, en San Pablo y en Temuco, consolidaron el trabajo conjunto y acercaron a través de sus profesores e investigadores a los países de la región, reforzando la iniciativa formadora de los Congresos de Anatomía del Cono Sur.

Finalmente, en 1999, después de un lapso de dos décadas, se retoma aquella necesidad de fomentar la anatomía y se crean los Congresos de Anatomía del Cono Sur, que tuvieron la aprobación de las respectivas comisiones directivas de la Sociedad Chilena de Anatomía (SChA) y de la Asociación Rioplatense de Anatomía (ARA). En el estatuto de ambas sociedades de anatomía se establece -en sus primeros artículosque deben tratar de mejorar los conocimientos de la anatomía en los ámbitos geográficos que representan. La propuesta de Mariano del Sol (Chile) y Ricardo Losardo (Argentina), con la creación de los Congresos del Cono Sur, cumplía con ello y reforzaba aquel objetivo, fortaleciendo la interrelación y la comunicación de los asociados entre ambos lados de la Cordillera de los Andes.

Es de resaltar que una década después de la creación de estos Congresos de Anatomía del Cono Sur, se refuerza el encuentro y acercamiento de los profesionales con el inicio de los SILAT (Simposios Ibero latinoamericanos de Terminología) extensión de la Asociación Panamericana de Anatomía (APA) y que repercutieron positivamente en la actividad científica de los países que participaban habitualmente en estos congresos del Sur de América (Cruz-Gutiérrez et al., 2010 b).

Los Congresos de Anatomía del Cono Sur se realizaron en forma conjunta con tres Congresos Panamericanos de 
Anatomía (XV, 2004; XVII, 2010; y XIX, 2019) y cuatro SILAT (V, 2010; XIII, 2015; XVI, 2018; y XVII, 2019), lo que demuestra la aceptación continental de este emprendimiento.

No podemos dejar de mencionar a la Revista Chilena de Anatomía, que tuvo un protagonismo particular en esta unión ya que en sus páginas se volcaron los resúmenes de los trabajos de los primeros tres Congresos de Anatomía del Cono Sur (publicados los años 2000, 2001 y 2002). Esta revista fue continuación de los Anales de Anatomía Normal de la Sociedad Chilena de Anatomía; y posteriormente se denominó International Journal of Morphology (revista donde se publicaron los resúmenes de los congresos IV al $\mathrm{X}$, entre los años 2003 y 2009). En 2010, Scientific Electronic Library Online (SciELO) dejó de incluir en su Base de Datos los resúmenes de los trabajos presentados en los diferentes eventos científicos de todas las áreas del conocimiento, y como consecuencia de esto, los resúmenes de los Congresos de Anatomía del Cono Sur ya no se volvieron a incluir Online. En el año 2010 y 2012 se publicaron solamente impresos los resúmenes de los trabajos presentados en el XII y XIII Congresos, respectivamente. Por otra parte, por razones de indexación y además, por los altos costos que significaba la impresión de la revista, a partir de 2013 los resúmenes de los congresos dejaron de ser impresos en International Journal of Morphology.

La Revista Chilena de Anatomía, a través de la iniciativa y gestión del Dr. Ricardo Losardo, fue también el órgano oficial de publicación de la nueva Asociación Argentina de Anatomía, desde sus inicios en el año 2000, siendo también una expresión de unión de ambos países cordilleranos y el punto de culminación del propósito de confraternidad de estos Congresos de Anatomistas del Cono Sur. International Journal of Morphology continuó siendo a partir de 2003, el Órgano Oficial de la Asociación Panamericana de Anatomía, Sociedad Chilena de Anatomía y Asociación Argentina de Anatomía.

\section{CONSIDERACIONES FINALES}

Estos Congresos de Anatomía del Cono Sur, creados al final del segundo milenio, con normativas consensuadas y con continuidad anual, lo hacen una entidad "de hecho". Este artículo constituye una manera de registrar su historia y de este modo recordar la trayectoria de estos 21 años de existencia.

Se realizaron de manera habitual en forma conjunta con las sociedades argentina, brasileña y chilena de anatomía, así como también con la Asociación Panamericana de Anatomía y la Academia Panamericana de Anatomía, a través de los Congresos Panamericanos de Anatomía y de los Simposios Íbero latinoamericanos de Terminología (SILAT), demostrando así su aprobación por los morfólogos internacionales.
Estos encuentros logran varios fines: a) permiten la superación científica de los asociados de ambas entidades trasandinas; b) fomentan el estudio e impulsaban la investigación de la anatomía; c) tratan de unificar los métodos de enseñanza universitaria; d) promueven el intercambio de conocimientos científicos entre sus concurrentes; y e) estrechan las relaciones humanas e institucionales entre ambas sociedades de anatomía.

DEL SOL, M. \& LOSARDO, R. J. Congresses of Anatomy of the Southern Cone. 21 years of history. Int. J. Morphol., 38 (3):689$705,2020$.

SUMMARY: We make a historical review of the Congresses of Anatomy of the Southern Cone. At the beginning of the year 1999, an agreement was established between the «Sociedad Chilena de Anatomía» and the «Asociación Rioplatense se Anatomía» to jointly conduct the scientific congresses of both societies. A few months later, also at the proposal of Drs. Mariano del Sol and Ricardo Losardo, a new entity was requested to be called initially "Congress of Anatomists of the Southern Cone", whose purpose would be to gather not only Argentine, Uruguayan and Chilean anatomists, but also incorporate morphologists from other countries in South America, including Brazilians and Paraguayans. In this way, the joint realization of the three congresses (Chilean, Rioplatense and the Southern Cone) began with a massive participation of the morphologists of numerous countries of our continent. The "Congresses of Anatomy of the Southern Cone" (so called since the II Congress held in Chile) have normally been held every year, often jointly with the Argentine, Chilean, Brazilian and Pan American Congresses of Anatomy, among other events. Upon the 21 st anniversary of the beginning of this fruitful and beneficial scientific union we wanted to capture in this article the origins of these Congresses of Anatomy of the Southern Cone, as well as the reasons that led to its creation. Likewise, the meetings held in the first 21 years (1999-2019) were listed, indicating their dates, headquarters and presidents.

KEY WORDS: Anatomy; Southern Cone Anatomy Congress; History of Anatomy; Anatomy Societies.

\section{REFERENCIAS BIBLIOGRÁFICAS}

Asociación Argentina de Anatomía. XLII Congreso Argentino de Anatomía, Mar del Plata, 20 - 21 de noviembre de 2005. Int. J. Morphol., 24 (Suppl. 1):114-21, 2006.

Asociación Argentina de Anatomía. XLIII Congreso Argentino de Anatomía, Rosario, 5 , 6 y 7 de octubre de 2006. Int. J. Morphol., 25 (1): 235 9, 2007. http://dx.doi.org/10.4067/S0717-95022007000100023

Campus tv Universidad de Talca. Inauguración del XXXIV Congreso de Anatomía en la Universidad de Talca, Chile, 20-23 de noviembre de 2013. https://www.youtube.com/watch?v=Bbgx_IcTVrM

Cárdenas, J. \& Rodríguez, A. La Sociedad Chilena de Anatomía. El inicio. Int. J. Morphol., 36(3):1075-8, 2018. 
Cruz-Gutiérrez, R.; Rodríguez-Torres, A.; Prates, J.C.; Losardo, R.J. \& Valverde Barbato de Prates, N. E. Simposios Ibero latinoamericanos de Terminología. Características Generales. Int. J. Morphol., 28(2):643-6, 2010.

Cruz-Gutiérrez, R.; Rodríguez-Torres, A.; Prates, J.C.; Losardo, R.J.; Valverde Barbato de Prates, N. E. Simposios Ibero latinoamericanos de Terminología Anatomía, Histología y Embriología. Int. J. Morphol., 28(1): 337-40, 2010.

Losardo, R. J. Asociación Panamericana de Anatomía. Reseña histórica y normativas vigentes. Int. J. Morphol., 27 (4):1345-52, 2009.

Losardo, R. J.; Cruz-Gutiérrez, R.; Prates, J. C.; Rodríguez-Torres, A.; Valverde-Barbato de Prates, N. E.; Arteaga-Martínez, M.; HaltíCabral, R. Alfonso Roque Albanese: pionero de la cirugía cardíaca latinoamericana. Homenaje de la Asociación Panamericana de Anatomía. Int. J. Morphol. 35 (3):1016-25, 2017.

Rodríguez, A. \& Cárdenas, J. Los orígenes de la Sociedad de Anatomía Normal y Patológica de Chile. Int. J. Morphol. 35(2):751-5, 2017.

Sigarán-Ramírez, M. F.; Cruz-Gutiérrez, R.; Yglesias-Vieto, Á. \& Losardo, R. J. Historia de la Asociación Centroamericana de Anatomía (1965-2014). Int. J. Morphol., 36(3):1130-3, 2018.

Sociedad Chilena de Anatomía \& Asociación Rioplatense de Anatomía. I Congreso de Anatomía del Cono Sur, XXXVI Congreso Rioplatense de Anatomía, XX Congreso Chileno de Anatomía, Mendoza, 7-8-9-10 de octubre de 1999. Rev. Chil. Anat., 18(1):125-214, 2000. http://dx.doi.org/10.4067/S0716-98682000000100017

Sociedad Chilena de Anatomía \& Asociación Rioplatense de Anatomía. II Congreso de Anatomía del Cono Sur, XXI Congreso Chileno de Anatomía, XXXVII Congreso Rioplatense de Anatomía, Santiago de Chile, 2-3-4 de noviembre de 2000. Rev. Chil. Anat., 19(1): 73-123, 2001. http://dx.doi.org/10.4067/S071698682001000100013

Sociedad Chilena de Anatomía \& Asociación Argentina de Anatomía. III Congreso de Anatomía del Cono Sur, XXXVIII Congreso Argentino de Anatomía, XXII Congreso Chileno de Anatomía, Mendoza, 7-8-9-10 de octubre de 1999. Rev. Chil. Anat., 20(1):69110, 2002. http://dx.doi.org/10.4067/S0716-98682002000100010

Sociedad Chilena de Anatomía \& Asociación Argentina de Anatomía. IV Congreso de Anatomía del Cono Sur, XX Congresso Brasileiro de Anatomia, XXIII Congreso Chileno de Anatomía, XXXIX Congreso Argentino de Anatomía y Primer Simpósio sobre Ensino de Anatomia, Maceió, Alagoas, Brasil, durante los días 6, 7, 8, 9, 10 y 11 de octubre de 2002. Int. J. Morphol., 21(1):49-95, 2003. http:// dx.doi.org/10.4067/S0717-95022003000100008

Sociedad Chilena de Anatomía \& Asociación Argentina de Anatomía. V Congreso de Anatomía del Cono Sur, XXIV Congreso Chileno de Anatomía, XL Congreso Argentino de Anatomía, Temuco, Chile, $12,13,14,15$ y 16 de noviembre de 2003. Int. J. Morphol., 22(1):35-101, 2004. http://dx.doi.org/10.4067/S071795022004000100006

Sociedad Chilena de Anatomía \& Asociación Argentina de Anatomía. Reunión de Integración de la Morfología Panamericana, XXV Congreso Chileno de Anatomía, XLI Congreso Argentino de Anatomía, XXI Congresso Brasileiro de Anatomia, VI Congreso de Anatomía del Cono Sur, XV Congreso Panamericano de Anatomía, II Simpósio sobre Ensino de Anatomia, Foz de Iguazú, Brasil y Puerto Iguazú, Argentina, 24, 25, 26, 27 y 28 de octubre de 2004. Int. J. Morphol., 23(1):45-94, 2005. http://dx.doi.org/10.4067/S071795022005000100009

Sociedad Chilena de Anatomía. XXVI Congreso Chileno de Anatomía y VII Congreso de Anatomía del Cono Sur, 12 al 15 de octubre de 2005. Int. J. Morphol., 24(Suppl. 1):5-113, 2006.

Sociedad Chilena de Anatomía. XXII Congresso Brasileiro de Anatomia, XXVII Congreso Chileno de Anatomía, VIII Congreso de Anatomía del Cono Sur. 8-12 de outubro de 2006, Florianópolis, Brasil. Int. J. Morphol., 25(1):145-234, 2007. http://dx.doi.org/10.4067/ S0717-95022007000100022
Sociedad Chilena de Anatomía. IX Congreso de Anatomía del Cono Sur, XXVIII Congreso Chileno de Anatomía, 29-31 de octubre de 2007, Talca, Chile. Int. J. Morphol., 26(1):170-226, 2008. http:// dx.doi.org/10.4067/S0717-95022008000100028

Sociedad Chilena de Anatomía. X Congreso de Anatomía del Cono Sur, XXIII Congresso Brasileiro de Anatomia, XI Congresso Luso-Brasileiro de Anatomia, XXVIII Congreso Chileno de Anatomía. 19-23 de octubre de 2008, Belém-Para-Brasil. Int. J. Morphol., 27(1):245-78, 2009. http://dx.doi.org/10.4067/S0717-95022009000100041

Sociedad Chilena de Anatomía. XVII Congreso Panamericano de Anatomía, XXII Congreso de Anatomía del Cono Sur, XXXI Congreso Chileno de Anatomía, V Simposio Ibero-Latinoamericano de Terminología Anatómica SILAT V. 25 al 30 de octubre de 2010, Temuco - Chile. Int. J. Morphol., 28(4):1327-415, 2010.

Sociedad Chilena de Anatomía. XIII Congreso de Anatomía del Cono Sur, XXXII Congreso Chileno de Anatomía, 27, 28 y 29 de octubre, Santa Marta, Colombia. Int. J. Morphol., 30(3):1203-35, 2012.

Talamoni, A. C. B. \& Bertolli Filho, C. A anatomia e o ensino de anatomia no Brasil: a escola Boveriana. Hist. Cien. Saúde-Manguinhos, 21(4):1301-22, 2014

\author{
Dirección para correspondencia: \\ Prof. Dr. Mariano del Sol \\ Centro de Excelencia en Estudios Morfológicos \\ y Quirúrgicos (CEMyQ) \\ Universidad de La Frontera \\ Temuco \\ CHILE
}

Email: mariano.delsol@ufrontera.cl

Recibido: 14-12-2019

Aceptado:12-01-2020 